ARTICLE

\title{
A tissue-bioengineering strategy for modeling rare human kidney diseases in vivo
}

J. O. R. Hernandez ${ }^{1,8}$, X. Wang ${ }^{1,8}$, M. Vazquez-Segoviano ${ }^{1}$, M. Lopez-Marfil (1) ${ }^{1}$, M. F. Sobral-Reyes ${ }^{1}$, A. Moran-Horowich', M. Sundberg (1) 2,3, D. O. Lopez-Cantu', C. K. Probst4,5, G. U. Ruiz-Esparza', K. Giannikou $3,4,5$, R. Abdi6, E. P. Henske $3,4,5$, D. J. Kwiatkowski (1) 3,4,5, M. Sahin (D) 2,3 \& D. R. Lemos (1) 1,3,7凶

The lack of animal models for some human diseases precludes our understanding of disease mechanisms and our ability to test prospective therapies in vivo. Generation of kidney organoids from Tuberous Sclerosis Complex (TSC) patient-derived-hiPSCs allows us to recapitulate a rare kidney tumor called angiomyolipoma (AML). Organoids derived from $\mathrm{TSC2}^{-/-}$hiPSCs but not from isogenic $\mathrm{TSC2}^{+/-}$or $\mathrm{TSC2}^{+/+}$hiPSCs share a common transcriptional signature and a myomelanocytic cell phenotype with kidney AMLs, and develop epithelial cysts, replicating two major TSC-associated kidney lesions driven by genetic mechanisms that cannot be consistently recapitulated with transgenic mice. Transplantation of multiple $\mathrm{TSC2}^{-/-}$renal organoids into the kidneys of immunodeficient rats allows us to model AML in vivo for the study of tumor mechanisms, and to test the efficacy of rapamycin-loaded nanoparticles as an approach to rapidly ablate AMLs. Collectively, our experimental approaches represent an innovative and scalable tissue-bioengineering strategy for modeling rare kidney disease in vivo.

\footnotetext{
${ }^{1}$ Renal Division, Brigham and Women's Hospital, Boston, MA 02115, USA. ${ }^{2}$ Rosamund Zander Stone Translational Neuroscience Center, Department of Neurology, Boston Children's Hospital, Boston, MA 02115, USA. ${ }^{3}$ Harvard Medical School, Boston, MA 02115, USA. ${ }^{4}$ Cancer Genetics Lab, Division of Pulmonary and Critical Care Medicine, Brigham and Women's Hospital, Boston, MA 02115, USA. ${ }^{5}$ Center for LAM Research and Clinical Care, Division of Pulmonary and Critical Care Medicine, Brigham and Women's Hospital, Boston, MA 02115, USA. ${ }^{6}$ Transplantation Research Center, Renal Division, Brigham and Women's Hospital, Harvard Medical School, Boston, MA, USA. ${ }^{7}$ Harvard Stem Cell Institute, Cambridge, MA 02138, USA. ${ }^{8}$ These authors contributed

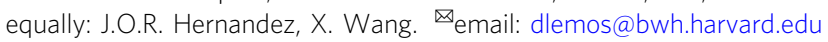


C ertain human diseases are driven by genetic and cellular mechanisms that cannot be recapitulated using transgenic mouse models. The lack of an in vivo model represents a limitation for the elucidation of disease mechanisms, and for testing prospective therapies on a preclinical level. One such disease is renal angiomyolipoma (AML), a tumor found in $80 \%$ of patients with Tuberous Sclerosis Complex (TSC). AML growth can cause kidney failure and lead to premature death due to the formation of vascular aneurysms prone to spontaneous bleeding $^{1,2}$. AMLs have been classified as perivascular epithelioid cell tumors, or PEComas, a term that designates mesenchymal neoplasms composed of anatomically and immunohistochemically distinctive myoid cells expressing smooth muscle and melanogenic genes ${ }^{3-6}$.

Rapamaycin analogs (rapalogs) are the main treatment for AML. Rapalogs reduce tumor size by inhibiting the dysregulated activity of mTORC1 that results from biallelic inactivation of TSC1 or TSC2 and loss of activity of the encoded proteins, namely TSC1 (hamartin) or TSC2 (tuberin), which act as repressors of the metabolic activator kinase $\mathrm{mTOR}^{7}$. In their current form, however, rapalog therapies rarely abrogate AML completely, making life-long treatment necessary. Relapse often occurs after treatment is interrupted due to adverse effects that may include proteinuria, oral ulcers, dyslipidemia and pneumonitis ${ }^{8-11}$. The lack of animal models has been an obstacle for the development of therapies and for the improvement of rapalog-based treatments.

The etiological mechanisms driving AML are difficult to recapitulate with transgenic animal models. AMLs are initiated by a Knudson's two-hit mechanism of tumorigenesis following a second copy neutral loss-of-heterozygosity ( $\mathrm{LOH}$ ) mutation in the TSC1 or, more commonly, in the TSC2 locus, in addition to a pre-existing germline inactivating mutation ${ }^{12,13}$. The second hit results in biallelic inactivation of either TSC1 or TSC2, and constitutive mTOR activation, driving anabolic cell metabolism and aberrant tissue growth ${ }^{14}$. Efforts to recapitulate these mechanisms in vivo have been challenging due to the fact that biallelic inactivation of TSC1 or TSC2 during development causes embryonic lethality. In addition, the developmental mechanisms giving raise to AML lesions has remained elusive ${ }^{4,15}$, contributing to the lack of success in previous attempts to ablate TSC1 or TSC2 by means of tissue-specific Cre-mediated recombination ${ }^{16,17}$. Limited success was achieved using an in vivo approach that involved low-dose doxycycline-induced DNA recombination events to ablate $T s c 1$ stochastically in mice carrying a ubiquitously expressed Cre transgenic allele ${ }^{18}$. Using this strategy, small kidney lesions with characteristics of AMLs could be detected, but the resulting number of mice carrying lesions was small, reducing the suitability of this model for either mechanistic or drug-testing studies $^{18}$.

In this work, we present a tissue-bioengineering approach for modeling TSC-associated human kidney diseases in vivo, using transplanted hiPSC-derived renal AML organoids. We show that nephric differentiation of patient-derived, and genetically-edited TSC2 $2^{-/-}$hiPSCs but not of isogenic TSC2 ${ }^{+/-}$hiPSCs $^{19}$, resulted in formation of two-dimensional (2D) and three-dimensional (3D) kidney tissues recapitulating TSC-associated AML and cystic disease. Transplantation of multiple 3D TSC2 $2^{-/-}$organoids into the kidneys of immunodeficient rats results in fully vascularized human AML xenografts that permitted us to identify tumor resistance mechanisms, and to test the efficacy of an in situ approach for rapid tumor ablation. The methodology presented here is broadly applicable for the study of other rare kidney diseases for which no in vivo experimental model currently exists.

\section{Results}

Nephric differentiation of $T S C 2^{-/-}$hiPSCs results in renal vesicle cultures containing myoid cells with a melanogenic phenotype. To understand how lack of TSC2 affects the early stages of nephric differentiation, we used an in vitro protocol previously developed for the directed differentiation of hiPSCs into kidney tissues ${ }^{20}$. We used a set of three isogenic hiPSC lines that included a line derived from an individual carrying a heterozygous 9 -bp deletion in the TSC2 locus $\left(T S C 2^{+1-}\right)$, a second isogenic TSC2 ${ }^{-1-}$ hiPSC line that was generated by introducing a TALEN-engineered second deletion in the wild type (WT) TSC2 allele of the patient-derived hiPSC line ${ }^{19}$, and lastly a $T S C 2^{+/+}$ hiPSC line in which the original TSC2 deletion present in the patient-derived hiPSC line was corrected using CRISPR-Cas9 (Fig. 1a) ${ }^{19}$. The TSC2 ${ }^{-1-}$ hiPSC line displayed significantly increased mTOR activity compared to the TSC $2^{+1-}$ and TSC $2^{+1+}$ hiPSC lines, as indicated by the levels of phosphorylation of the ribosomal protein S6 (Phospho-S6) detected by Western Blot (Fig. 1b). No differences in phospho-S6 levels were observed between the $T S C 2^{+/-}$and TSC2 $2^{+/+}$hiPSC lines, a finding that was consistent with the idea that one functional TSC2 allele is sufficient to preserve mTOR activity under these conditions (Fig. 1b).

To generate renal tissues from the three hiPSC lines in $2 \mathrm{D}$ conditions, we used a previously established protocol that involves an initial step of metanephric mesenchyme induction by initial incubation with the WNT activator CHIR99201 (CHIR) at $8 \mu \mathrm{M}$ for 4 days, followed by incubation with $20 \mathrm{ng} / \mathrm{ml}$ Activin A for three days (Fig. 1c) and subsequent incubation with $25 \mathrm{ng} / \mathrm{ml}$ fibroblast growth factor 9 (FGF9) for two days ${ }^{20}$. On Day 9, the $2 \mathrm{D}$ cultures received a $1-\mathrm{h}$ pulse of $3 \mu \mathrm{M}$ CHIR. The $8-\mu \mathrm{M}$ concentration of CHIR during the initial induction phase was chosen based on comparable mRNA expression profiles observed for the nephric lineage homeobox genes SIX2 and SALL1 in the three hiPSC genotypes between Day 0 and Day 9, which were determined by quantitative polymerase chain reaction (qPCR) (Fig. 1d). Both SIX2 and SALL1 were also detected by immunofluorescence in cultures of the three hiPSC lines, confirming induction of these nephric lineage markers (Supplementary Fig. 1a). Microscopic field quantification indicated that $\sim 82,85$, and $91 \%$ of $T S C 2^{+/-}, \mathrm{TSC}^{-1-}$, and $\mathrm{TSC} 2^{+/+}$cells, respectively, were $\mathrm{SIX}_{2}^{+}$; and $\sim 80,83$, and $87 \%$ of $\mathrm{TSC}^{+/-}$, $\mathrm{TSC}^{-/-}$, and $\mathrm{TSC}^{+/+}$cells, respectively, were SALL1 ${ }^{+}$, indicating efficient induction of all three cell lines (Supplementary Fig. 1a). After confirming induction of metanephric mesenchyme from the three hiPSC lines, we assessed their ability to subsequently form the renal vesicles that give rise to nephrons. To that end, we continued incubating the metanephric mesenchyme cultures with FGF9 until Day 14. During this stage, the cultures were exposed to a $24-\mathrm{h}$ pulse of $1 \mu \mathrm{M}$ retinoic acid on Day 13 of differentiation (Fig. 1c). This modification to the original protocol was adopted from a recent protocol for the differentiation of podocytes ${ }^{21}$ and was introduced based on our observation of glomeruli of an increased size present in Day 21 cell cultures (Supplementary Fig. 1b, c). In order to assess the formation of renal vesicles on Day 14 we used a combination of immunostaining for the homeobox transcription factor PAX8 and qPCRs to detect the expression of the nephric homeobox genes PAX8, $L H X 1, H N F 1$, and WT1. We confirmed the formation of cell aggregates with the morphology of renal vesicles containing $\mathrm{PAX}^{+}$renal progenitor cells (Fig. 1f). In addition, we detected similar expression levels for renal vesicle markers in $T S C 2^{-1-}$, $\mathrm{TSC}_{2}{ }^{+-}$, and $\mathrm{TSC} 2^{+/+}$cell cultures, and found that all were upregulated compared to undifferentiated hiPSC cultures (Fig. 1g). 


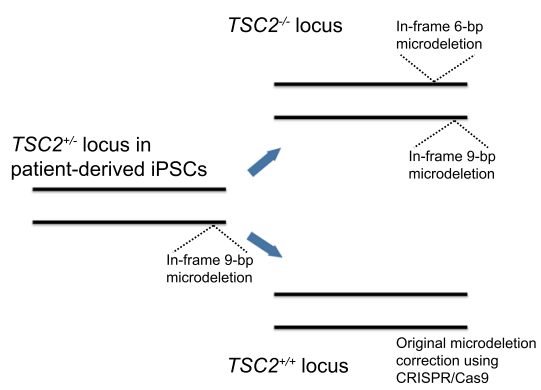

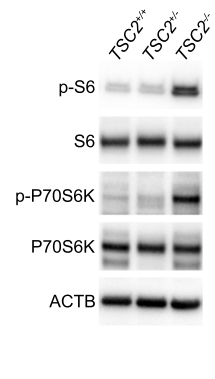

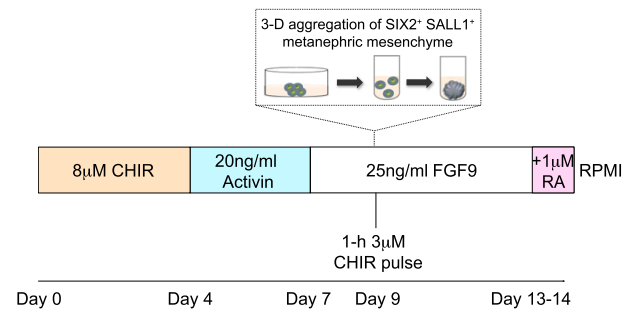

d

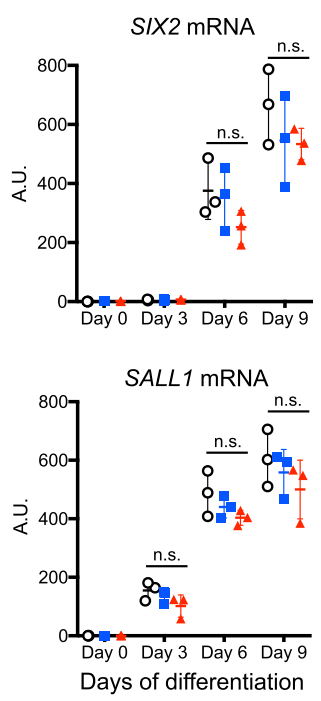

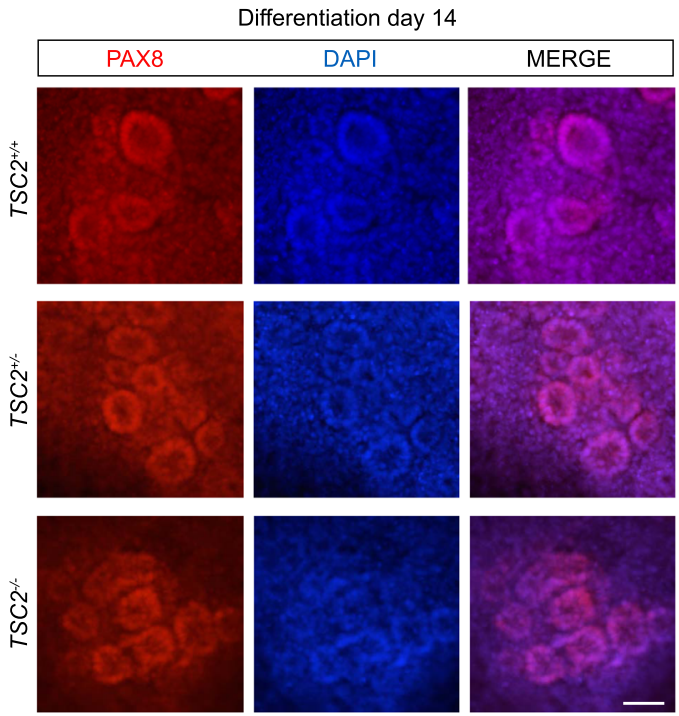

$\mathbf{f}$

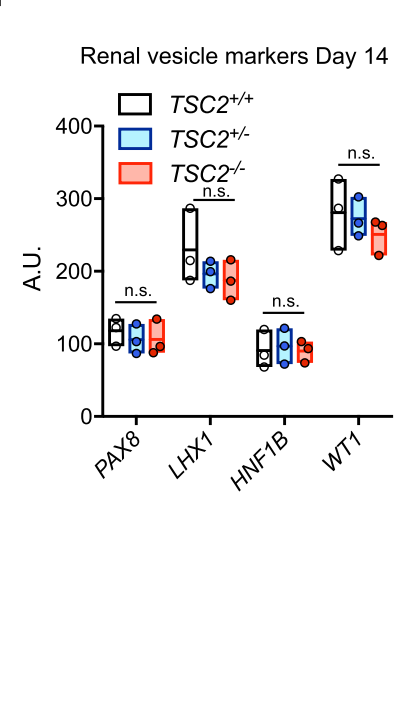

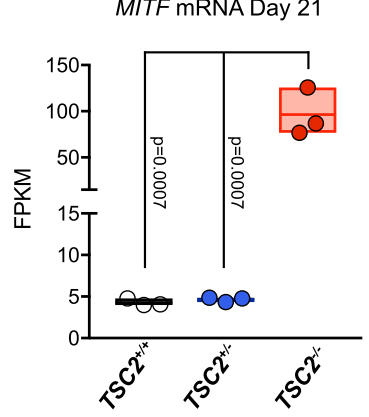

h

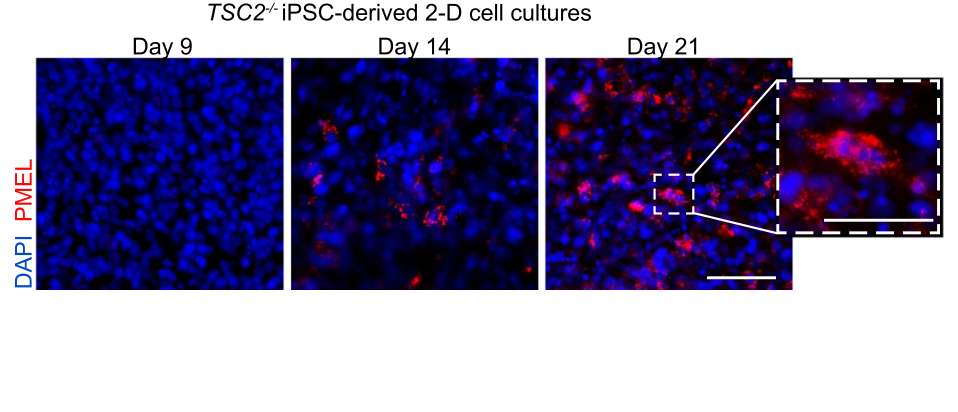

$\mathrm{PMEL}^{+}$cells

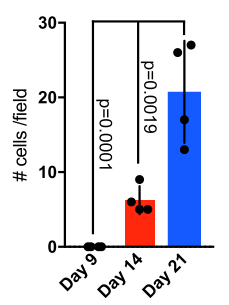

Given that loss of TSC1 or TSC2 is a common occurrence in kidney AMLs from TSC patients ${ }^{13}$, we next performed global gene expression analysis comparison by means of whole transcriptome RNA sequencing (RNA-seq) of TSC2 $2^{+/-}, \mathrm{TSC}^{+/+}$, and $T S C 2^{-1-}$ iPSC-derived organoids generated by differentiation of Day- 9 metanephric mesenchymal cell aggregates in three-dimensional culture conditions ${ }^{22}$. Our analysis revealed that the AML master driver oncogene $M I T F^{23}$ was significantly upregulated in $T S C 2^{-1-}$ iPSC-derived Day-21 organoids, compared to $\mathrm{TSC}^{+/-}$and TSC2 ${ }^{+/+}$iPSC-derived organoids (Fig. $1 \mathrm{~g}$ ). Using the mouse monoclonal antibody HMB45 which is routinely used in clinical histopathology for diagnosing $\mathrm{AML}^{5,24}$, we detected cells expressing the melanogenic protein premelanosome 17 (PMEL) in differentiating 2D cultures of TSC $2^{-1-}$ iPSC-derived cells but not in $\mathrm{TSC}^{+/-}{ }^{+1}$, TSC2 $2^{+/+}$iPSC-derived cell cultures (Fig. 1h). We first detected TSC2 $^{-/-}$PMEL-expressing cells on differentiation Day
14 and determined that their number increased by Day 21 (Fig. 1i). Of note, TSC2 ${ }^{-1-} \mathrm{PMEL}^{+}$cells had a plump myoid morphology (Fig. 1i), distinct from the typical dendritic morphology of epidermal melanocytes in culture ${ }^{25,26}$.

Taken together, these results indicated that while loss of TSC2 is not an impediment for the nephric specification of iPSCs into metanephric mesenchyme and formation of renal vesicles, the differentiation results in the generation of cells resembling the myoid melanogenic phenotype observed in kidney AML cells ${ }^{3,24}$

TSC2 ${ }^{-/-}$hiPSCs generate 3D renal organoids with characteristics of kidney AMLs in vitro. Our results indicating the specification of cells with myoid/ melanocytic characteristics throughout our protocol of nephric differentiation prompted us to investigate kidney AML features in $T S C 2^{-1-}$ iPSC-derived 
Fig. 1 Nephric differentiation of TSC2 ${ }^{-1}-$ hiPSCs results in generation of myoid melanocyte-like cells. a Schematic representation of the gene-editing

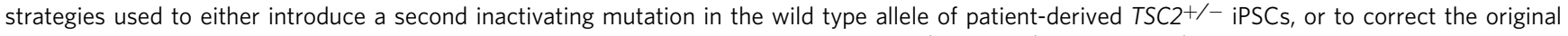
deletion mutation. $\mathbf{b}$ Immunoblots showing phosphorylation of $S 6$ and p-70S6K in TSC2 $+/+, T S C 2^{-/-}$, and TSC2 $/ /-$hiPSCs, indicating mTORC1 activation in the $\mathrm{TSC2}^{-/-}$cells. Similar results were obtained in two independent experiments. c Schematic representation for the hiPSC nephric differentiation protocol used to generate 2D renal tissues and 3D renal organoids. CHIR: CHIR99021; FGF9: fibroblast growth factor 9. d Quantitative real-timepolymerase chain reaction (RT-PCR) analysis of SIX2 and SALL1 mRNAs during the phase of metanephric mesenchyme induction. Curve points represent mean \pm SD in arbitrary units (A.U. normalized to $\beta$-actin), $n=3$ independent technical replicates. Nonsignificant mean differences determined by two-way ANOVA analysis using Tukey test for multiple comparisons are indicated as n.s. e Representative immunofluorescence images showing PAX8 expression in 2D TSC2 $+/+, \mathrm{TSC2}^{-/-}$, and TSC2 $+/-$cell cultures on Day 14 of differentiation. Scale bar, $50 \mu \mathrm{m}$. $\mathbf{f}$ Quantitative RT-PCR analysis of $P A X 8, L H X 1, H N F 1 B$ and WT1 mRNAs in 2D TSC2 ${ }^{+/+}, \mathrm{TSC2}^{-/-}$and TSC2 ${ }^{+/-}$cell cultures on differentiation Day 14, showing similar expression independent of genotype. Floating bars graph represent mean $\pm \mathrm{SD}, n=3$ independent experiments. Nonsignificant mean differences determined by two-way ANOVA analysis using Tukey test for multiple comparisons are indicated as n.s. $\mathbf{g}$ Expression of the AML transcription factor MITF as determined by RNAseq analysis of TSC2 $+/+$, $\mathrm{TSC2}^{+/-}$, and $\mathrm{TSC2}^{-/-}$iPSC-derived renal organoids on differentiation Day 21. Floating bar graphs represent mean $\pm \mathrm{SD}, n=3$ samples for each genotype, five organoids per sample. Gene expression is shown in fragments per kilo base per million mapped reads (FPKM) values. $P$ values for individual comparisons done using Tukey test in one-way ANOVA analysis are indicated. $\mathbf{h}$ Representative immunofluorescence images of 2D TSC2-/- iPSC-derived cell cultures showing expression of PMEL on differentiation Days 9, 14, and 21. Scale bars, $25 \mu \mathrm{m}$ and $12.5 \mu \mathrm{m}$ for high magnification image. i Bar graphs showing the quantification of $\mathrm{PMEL}^{+}$cells in immunofluorescence images taken at Days 9, 14, and 21 of differentiation in 2D conditions. Values in the bar graph represent mean $\pm S D, n=4$ experiments. $P$ values for individual comparisons done using Tukey test in one-way ANOVA analysis are indicated.

organoids. Our immunofluorescence analysis did not detect $\mathrm{PMEL}^{+}$cells in TSC2 $2^{+/-}$and $\mathrm{TSC2}^{+/+}$kidney organoids (Fig. 2d), a finding that was also consistent with a previous study using the Morizane protocol for nephric differentiation of hPSCs ${ }^{27}$. By contrast, and consistent with our analysis of Day-21 2D TSC2 ${ }^{-1-}$ cell cultures, we identified a large population of $\mathrm{PMEL}^{+}$cells in $\mathrm{TSC}^{-/-}$organoids, covering an area that was approximately $6.2 \%$ of the organoid sections analyzed (Fig. 2a). $\mathrm{PMEL}^{+}$cells were present in the interstitial space, but were not peritubular, a characteristic that distinguished them from renal fibroblasts observed in both human kidneys and hPSC-derived organoids. Similar to what we observed in our $2 \mathrm{D}$ cultures, $3 \mathrm{D}$ cultured $\mathrm{PMEL}^{+}$cells had a myoid spindle-like morphology with a granular staining pattern that resembled the characteristic phenotype of human kidney AML cells (Fig. 2a, Supplementary Fig. 2a) ${ }^{3}$. Co-expression of PMEL and glycoprotein NMB (GPNMB), another AML cell marker ${ }^{28}$, further confirmed the melanogenic phenotype of $T S C 2^{-1-}$ organoid cells (Fig. 2b). Next, we investigated the expression and distribution of cells expressing actin alpha 2, smooth muscle (ACTA2, commonly known as alpha-smooth muscle actin), whose aberrantly high expression is a hallmark of $\mathrm{AML}^{3}$, and which is expressed at low levels in non-injured kidneys and WT organoids ${ }^{29}$. We observed that ACTA2 was highly expressed in TSC2 ${ }^{-1-}$ organoids, with ACTA2 $^{+}$cells covering an area that represented $21.7 \%$ of the sections analyzed, compared to 3.28 and $3.7 \%$ of the areas in $\mathrm{TSC}^{+/+}$and $\mathrm{TSC}^{+/-}$organoid sections, respectively (Fig. 2c). Similar to what we observed for $\mathrm{PMEL}^{+}$cells, ACTA2 ${ }^{+}$cells were sparsely distributed throughout the stroma of $\mathrm{TSC}^{-1-}$ organoids (Fig. 2c). Consistent with what has been previously reported, the $\mathrm{ACTA}^{+}$cells observed in $\mathrm{TSC}^{+/-}$and $\mathrm{TSC}^{+/+}$organoids were mostly confined to regions surrounding nephron epithelial structures (Supplementary Fig. 2b). Morphologically, ACTA2 ${ }^{+}$ cells found in TSC2 $2^{-1-}$ organoids had a rounder and myoid morphology that best matched the well-characterized morphology of $\mathrm{ACTA}^{+}$cells observed in kidney AMLs (Fig. 2c, Supplementary Fig. $2 \mathrm{a})^{15}$. By contrast, ACTA2 ${ }^{+}$cells in $\mathrm{TSC2}^{+l-}$ and $\mathrm{TSC}^{+/+}$organoids presented an elongated spindle-like cell body with extended body processes resembling the morphology of kidney fibroblasts (Supplementary Fig. $2 \mathrm{~b})^{29}$. Given that coexpression of melanogenic and mesenchymal cell genes is unique to AML cells, we next investigated whether $T S C 2^{-1-}$ organoid cells had a myomelanocytic phenotype. Using immunofluorescence we were able to establish that $~ 94 \%$ of GPNMBexpressing cells were ACTA2 ${ }^{+}$(Fig. 2d). Cells co-expressing both markers were also detected in AML samples, but not in activated fibroblasts/myofibroblasts from injured $\mathrm{TSC}^{+/+}$organoids. In order to validate the myomelanocytic cell phenotype using a different technical approach, we analyzed the distribution of $\mathrm{PMEL}^{+}$and $\mathrm{ACTA}_{2}^{+}$cell populations in $\mathrm{TSC}^{-1-}$ organoids by means of fluorescence-activated cell sorting (FACs). Our analysis identified a population of $\mathrm{ACTA}^{+}$cells that represented approximately $31.2 \%$ of the total cells recorded in $\mathrm{TSC}^{-1-}$ organoid samples (Fig. 2e, Supplementary Fig. 3a). The same cell population constituted 3.5 and $4.1 \%$ of the cells recorded in $\mathrm{TSC}^{+/-}$and $\mathrm{TSC} 2^{+/+}$organoid samples, respectively (Fig. 2e). Within the ACTA2 ${ }^{+}$cell population, approximately $24.3 \%$ of the cells also expressed PMEL (Fig. 2e), whereas no PMEL ${ }^{+} \mathrm{ACTA2}^{-}$ cells were detected, a finding that was consistent with our immunofluorescence results for GPNMB and ACTA2 (Fig. 2d). Immunofluorescence tracking experiments in $2 \mathrm{D}$ cultures further indicated that $T S C 2^{-1-}$ myomelanocytic cells appeared by Day 14 of iPSC differentiation coinciding with the time of formation of renal vesicles (Supplementary Fig. 2c). We next compared the expression of myomelanocytic cell markers in prospectively purified live $\mathrm{GPNMB}^{+}$cells versus $\mathrm{GPNMB}^{-}$cell fractions of $\mathrm{TSC}^{-1-}$ organoids (Figure f, Supplementary Fig. 3b). We detected significant expression of AML markers MLANA, GPNMB, and CTSK in the GPNMB ${ }^{+}$fraction but not in the GPNMB $^{-}$fraction (Figure f). Expression of ACTA2 was detected in both cell populations, albeit more abundantly in the GPNMB cell fraction (Figure f), a result that was consistent with our FACs analysis results of PMEL and ACTA cell fractions. Taken together, our histology, FACS and gene expression data identified a population of $\mathrm{TSC}^{-1-}$ organoid cells that recapitulates the unique phenotype and gene expression profile observed in kidney AML myomelanocytic cells ${ }^{15}$. Additionally, we confirmed the abnormal expression of GPNMB and ACTA2 in $\mathrm{TSC2}^{-1-}$ organoids by means of Western Blot performed on protein extracts from $T S C 2^{+/-}$and $\mathrm{TSC}^{+/+}$and $T S C 2^{-1-}$ organoids (Fig. $2 \mathrm{~g}$ ).

Collectively these in vitro data indicate that renal organoids derived from $T S C 2^{-l-}$ hiPSCs, but not from TSC2 $2^{+/-}$hiPSCs, possessed several key characteristics of renal AML tumors, including the presence of myomelanocytic cells with a typical AML morphology. These results also provide critical experimental support to the hypothesis that the signals driving AML are present during renal development.

Gene expression analysis of $\mathrm{TSC2}^{-/-}$renal organoids validates an AML identity. To investigate how loss of TSC2 affects global gene expression in hiPSC-derived renal organoids, we performed pairwise DESeq2 differential gene expression analyses of our whole 


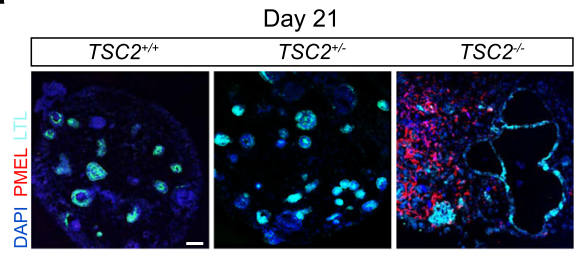

c

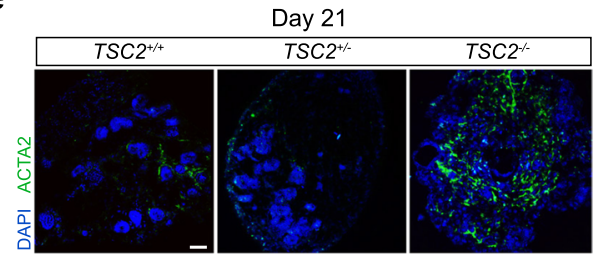

$\mathbf{e}$
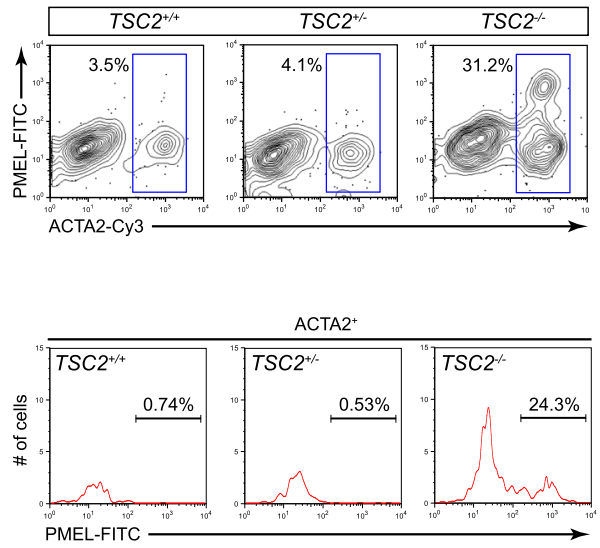

$\mathrm{PMEL}^{+}$area

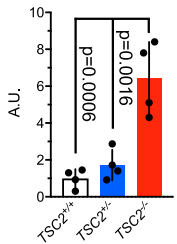

$\mathrm{ACTA}^{+}$area

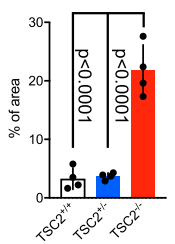

ACTA $2^{+}$cells

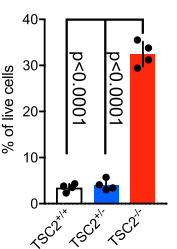

$\mathrm{PMEL}^{+}$cells

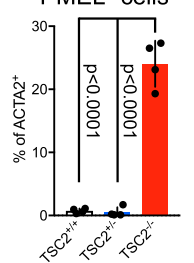

b

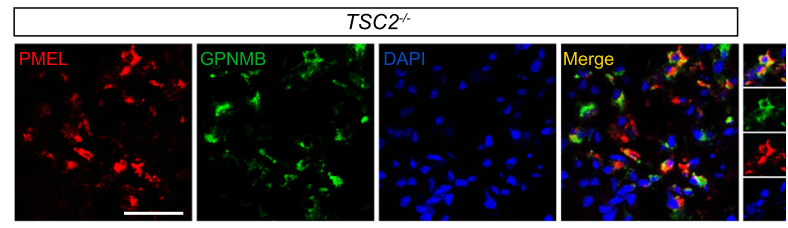

d
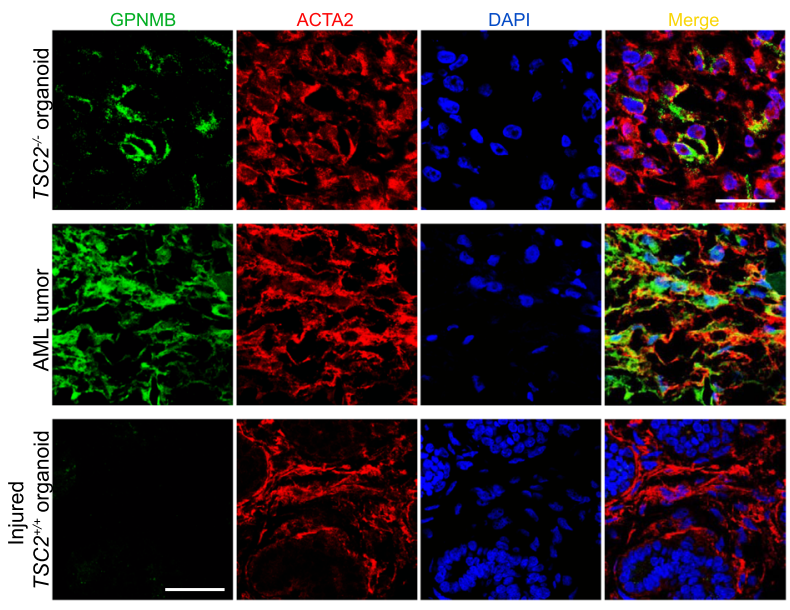

$\mathbf{f}$

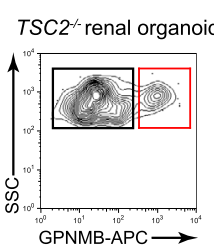

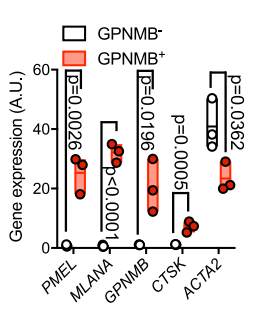

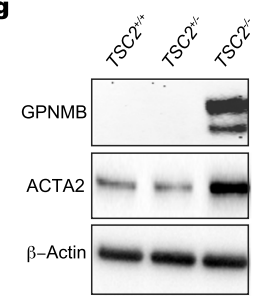

Fig. 2 TSC2 $^{-/-}$renal organoids recapitulate human AML tumor phenotype. a-c Confocal optical sections showing LTL with PMEL (a), PMEL and GPNMB (b), and ACTA2 (c) in Day $213 \mathrm{D}$ renal organoids derived from TSC2 $+/+, \mathrm{TSC2}^{-/-}$and TSC2 ${ }^{+/-}$hiPSCs. Bar graphs in (a) and (c) represent mean $\pm S D, n=4$ experiments. $P$ values for individual comparisons done using Tukey test in one-way ANOVA analysis are indicated. d Representative confocal immunofluorescence sections showing expression of GPNMB and ACTA2 in Day-21 TSC2 ${ }^{-/-}$renal organoids, kidney AML tumor and fibrotic Day-28 TSC $^{+/+}$renal organoids injured by incubation with interleukin $1 \beta$ for $96 \mathrm{~h}$. e Representative FACs plots and quantification showing the percentage of $\mathrm{ACTA}^{+}$cells and PMEL ${ }^{+}$cells in Day- $21 \mathrm{TSC2}^{+/+}, \mathrm{TSC2}^{-/-}$and $\mathrm{TSC2}^{+/-}$renal organoids. Bar graphs represent mean $\pm \mathrm{SD}, n=4$ experiments. $P$ values for individual comparisons done using Tukey test in one-way ANOVA analysis are indicated. $\mathbf{f}$ Representative cell sorting plot showing the GPNMB + (red square) and GPNMB- (black square) cell fractions in TSC2-/- renal organoids and gene expression levels for PMEL, MLANA, GPNMB, CTSK, and ACTA2, determined by Quantitative RT-PCR analysis. Floating bars graph represent mean $\pm S D, n=3$ independent experiments, containing three organoids each. $P$ values for individual comparisons done using two-tailed Student's t test are indicated. $\mathbf{g}$ Representative immunoblot showing expression of GPNMB and ACTA2 in renal organoids from the three genotypes. Two independent experiments were performed with similar results. Scale bars, $50 \mu \mathrm{m}$.

transcriptome RNA-seq datasets, comparing TSC2-1- organoids to $\mathrm{TSC}^{+/+}$and TSC2 ${ }^{+/-}$organoids, using Qlucore software. Several hundred genes were upregulated or downregulated at a false discovery rate $(\mathrm{FDR}) / q$ value $<0.05, p$ value $<0.002, \mid \log _{2}$ fold $\mid>2$ and $\mid \log _{2}$ fold change $\mid<-2$ (Supplementary Table 1). Among the genes upregulated in the TSC2 $2^{-1-}$ organoids were: MLANA, PMEL, GPNMB, CTSK, and ACTA2, with median expression fold change $121 \mathrm{x}-, 88 \mathrm{x}-, 34 \mathrm{x}-, 14 \mathrm{x}-, 9.5 \mathrm{x}$, respectively, all of which are known to be highly expressed in the renal angiomyolipoma (Fig. 3a). In contrast, DEseq 2 analysis of $T S C 2^{+/+} v s$. TSC $2^{+/-}$renal organoids did not reveal remarkable differences. Multiple housekeeping genes, including GPDH, GUSB, RPL19, showed no significant difference or minimal changes in expression across the three kidney organoid genotypes providing further confirmation that the changes observed were specific to the genes identified (Supplementary Fig. 4a).

Principal Component Analysis (PCA) of RNA-Seq data for all three genotypes also demonstrated that the $T S C 2^{-1-}$ organoids were transcriptionally different from $\mathrm{TSC}_{2}^{+/+}$and $\mathrm{TSC}^{+/-}$ kidney organoids, which clustered together (Fig. 3b). Hierarchical clustering using Spearman rank correlation for the 3000 most variable genes also demonstrated that the $T S C 2^{-/-}$organoids were distinct from the other two genotypes, with the latter appearing similar (Fig. 3c). Gene set enrichment analysis (GSEA) was performed comparing $T S C 2^{-/-}$renal organoid RNA data to that of $\mathrm{TSC}^{+/+}$renal organoids, and showed enrichment in multiple gene sets at $\mathrm{FDR} / \mathrm{q}<0.25$, with similar results in comparison of $T S C 2^{-/-}$and TSC $2^{+/-}$renal organoids. Hallmark gene sets enriched in expression in $\mathrm{TSC}^{-/-}$renal organoids in each comparison included IL6-JAK-STAT3 signaling, adipogenesis, angiogenesis, fatty acid metabolism, KRAS signaling, and estrogen response (Fig. 3d, Supplementary Fig. 4a, b).

We then investigated the similarity between the genes identified in the comparisons between TSC2-/- vs. TSC $2^{+/+}$ organoids against differential genes identified in the comparison between kidney AML vs. normal kidney (Fig. 3e). The 187 common differentially expressed genes included the AML 

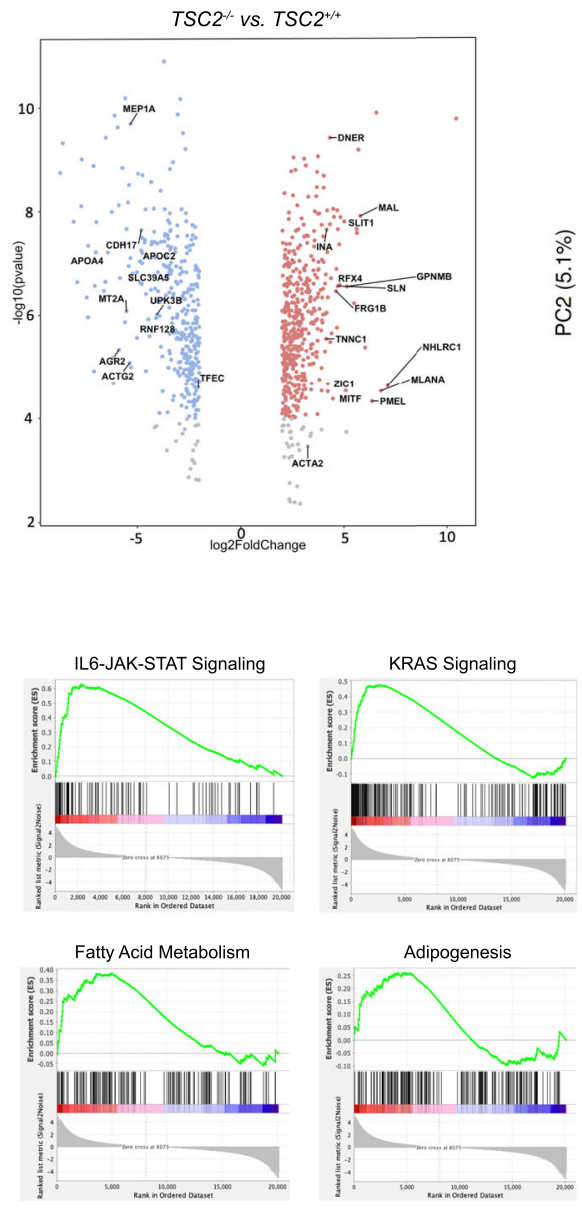

f
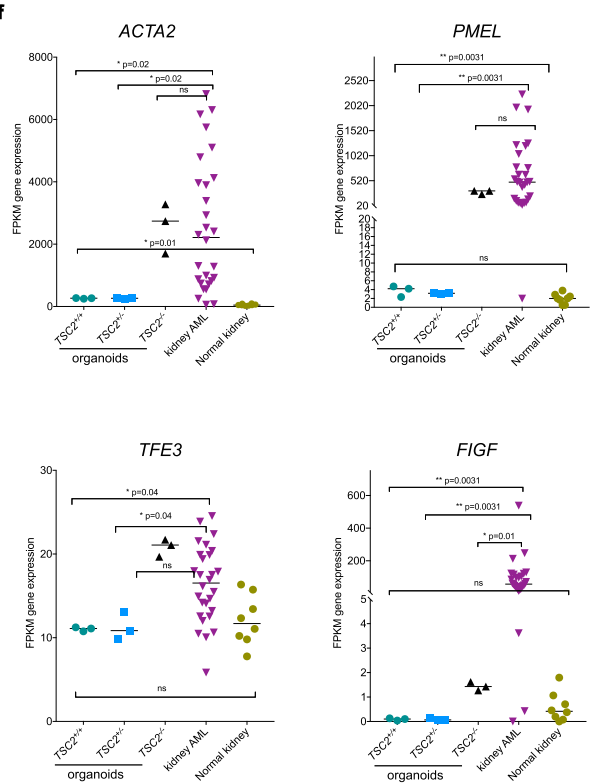

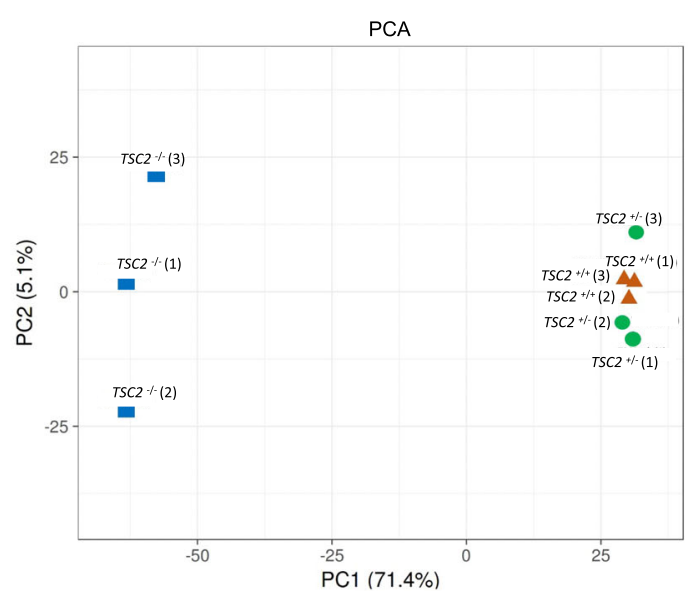

e
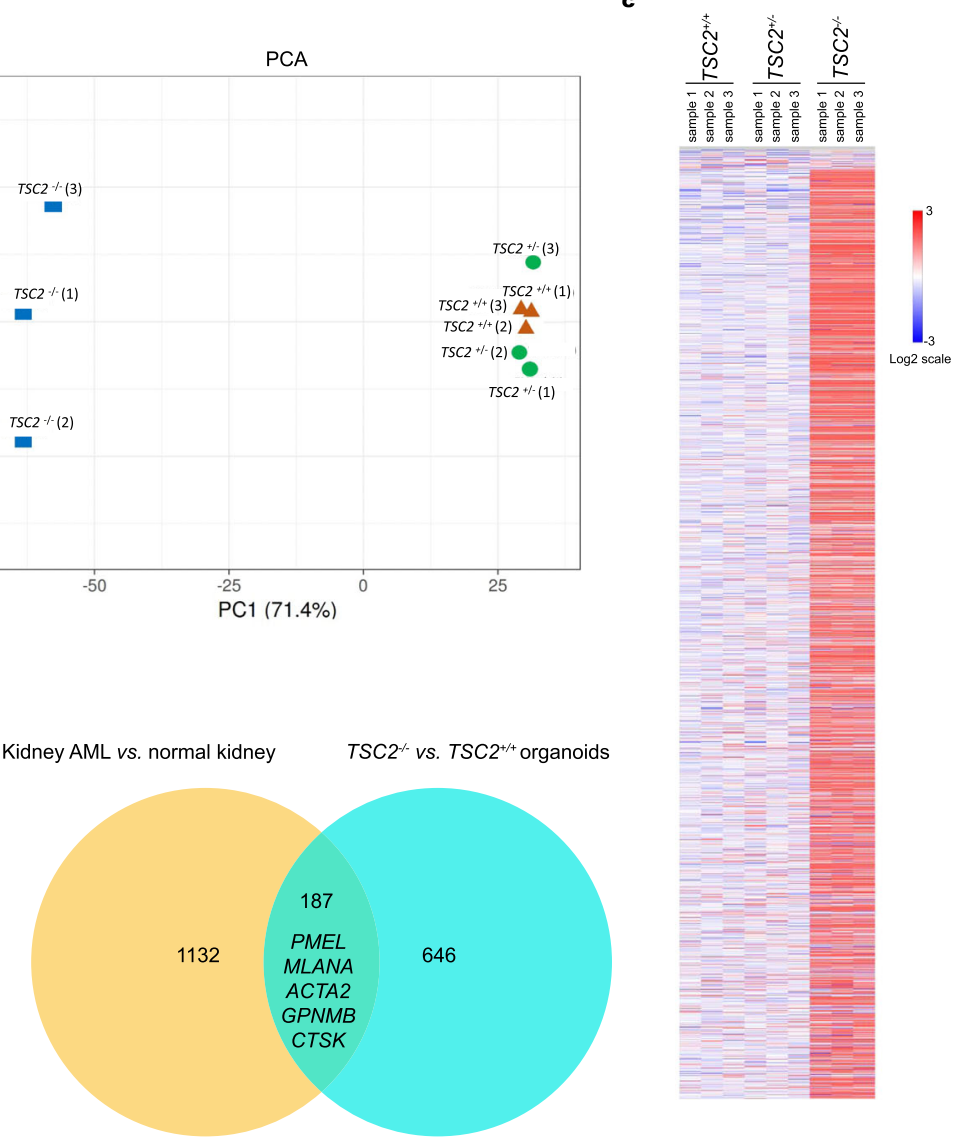
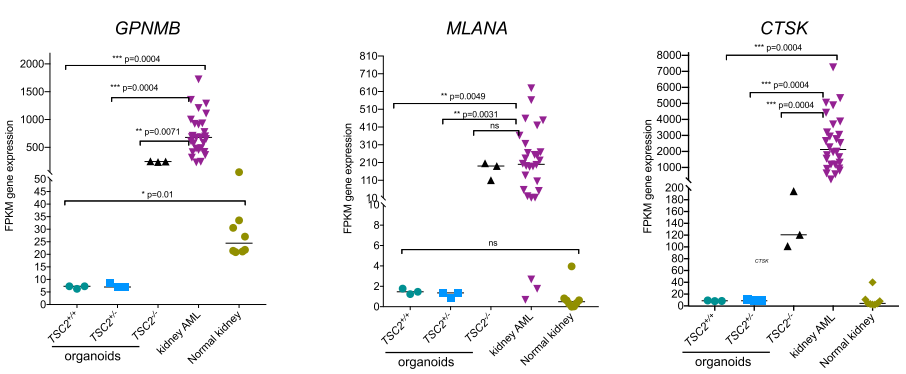

g

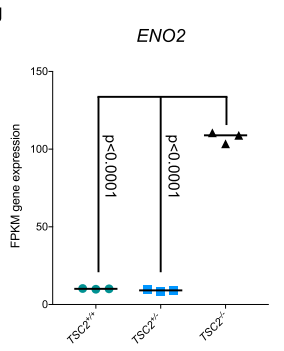

Enolase 2 content

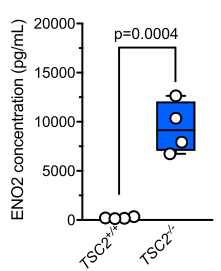

Enolase activity

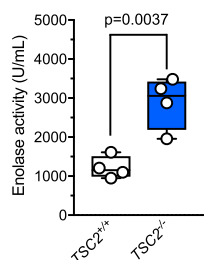

hallmark genes MLANA, PMEL, GPNMB, MITF, CTSK, and ACTA2, expanding the similarities observed between TSC2 ${ }^{-1-}$ organoids and kidney AML to other signature markers (Fig. 3e). Direct comparison of mRNA expression levels for those markers plus the AML transcription factors TFE3 and FIGF (Fig. 3f) confirmed a similar expression profile for $\mathrm{TSC}^{-/-}$organoids and kidney AML, compared to isogenic control organoids and to normal kidneys, respectively (Fig. 3f).

We next focused on enolase 2 (ENO2), a glycolysis enzyme upregulated in a variety of neuroendocrine tumors, including kidney AML (Supplementary Fig. 4d) ${ }^{30-32}$. We observed that ENO2 mRNA expression was significantly higher in $\mathrm{TSC}^{-1-}$ organoids compared to $\mathrm{TSC}^{+/+}$organoids (Fig. $3 \mathrm{f}$ ), a result that 
Fig. 3 Gene expression analysis of $\mathbf{T S C 2}^{-} /-, \mathbf{T S C 2}^{+/-}$, and $\mathbf{T S C 2}^{+/+}$renal organoids. a Volcano plots showing the distribution of all differentially expressed genes (DEGs) in TSC2 $2^{-/-}$renal organoids compared to $\mathrm{TSC2}^{+/+}$(left) and $\mathrm{TSC2}^{+/-}$(right) renal organoids $(F D R<0.05)$. Each dot represents a unique gene; red denotes $\log _{2}$ (fold change) $>2$, upregulated genes in TSC2-/-; blue denotes $\log _{2}$ (fold change) $<-2$, downregulated in TSC2 $2^{-/-}$. Selected statistically significant upregulated and downregulated genes (NCBI/Entrez names) are indicated, as determined by a two-sided Chi-Square test. b Principal Component Analysis (PCA) of RNA-Seq data from renal organoids of the three genotypes, $n=3$ samples for each genotype, five organoids per sample. c Heatmap showing hierarchical clustering of three different genotypes of kidney organoids using the top 3000 most variable genes. Color scale representative of gene expression level: red denotes $\log _{2} \leq 3$, blue denotes $\log _{2} \geq-3$. $\mathbf{d}$ Representative enrichment plots corresponding to gene set enrichment analysis (GSEA) for pairwise comparison of TSC2 $-/-$ vs. SSC2 $^{+/-}$. e Venn diagrams indicating 187 common differentially expressed genes, including signature AML markers, in $T S C 2^{-/-}$vs. TSC2 $2^{+/+}$renal organoids and kidney AML vs. normal kidney. $\mathbf{f}$ Comparative mRNA expression levels for AML hallmark genes in $\mathrm{TSC2}^{-/-}, \mathrm{TSC2}^{+/+}$, and $\mathrm{TSC2}^{+/-}$renal organoids ( $n=3$ each) compared to human kidney $\mathrm{AML}(n=28)$ and human kidney $(n=8) . P$ values for individual comparisons done using a two-sided Mann-Whitney $U$ test are indicated. Gene expression is shown in FPKM values. g Comparative ENO2 mRNA expression levels in TSC2 ${ }^{+/}$and $\mathrm{TSC2}^{+/-}, \mathrm{TSC}^{-/-}$renal organoids $(n=3$ each). $P$ values for the indicated individual comparisons done using two-tailed Student's $t$ test are shown. Gene expression is shown in FPKM values. $\mathbf{h}$, $\mathbf{i}$ Box-and-whisker plot showing minimum value, first quartile, median, third quartile and maximum value for ENO2 content (g) and for enolase activity (h) in whole extracts of TSC2 ${ }^{+/+}$and TSC2 $^{-} /-$ renal organoids. $P$ value for the 2 -tailed Student's $t$ test comparing $\mathrm{TSC2}^{-/-}$versus $T S C 2^{+/+}$is shown. $n=4$ independent experiments, containing three organoids each.

was consistent with both increased ENO2 protein levels (Fig. 3g) and increased overall enolase activity in $T S C 2^{-/-}$organoids (Fig. 3g).

Taken together, the common patterns of expression for hallmark genes and signaling pathways further reinforced the similarities between $T S C 2^{-1-}$ organoids and kidney AML, on a molecular level.

TSC2 inactivation drives renal organoid tubule cyst formation. Based on the dilated appearance of putative proximal tubules in TSC2 ${ }^{-1}-3 \mathrm{D}$ organoids (Fig. 2a), we set out to study the phenotype of TSC2 $2^{-/}$nephrons. We noted that brightfield imaging of Day-21 2D TSC2 ${ }^{+/+}$and TSC2 ${ }^{+/-}$cell cultures contained tubule like structures, whereas cultures derived from $T_{S C} 2^{-1-}$ hiPSCs showed cavitated structures resembling cysts (Fig. 4a). Because cysts are another common abnormality found in TSC patients with renal manifestations ${ }^{33}$, we investigated the cellular composition of the cysts to determine whether they were formed by tubule epithelial cells (TECs) derived from TSC $^{-/}-$hiPSCs. By means of immunofluorescence, we detected nephron segments corresponding to distal tubules expressing cadherin 1 (CDH1), proximal tubules containing brush borders labeled by lotus tetranoglobus lectin (LTL) and glomeruli containing podocytes expressing podocalyxin 1 (PODXL1) in the cell aggregates derived from $T S C 2^{+/+}$and TSC2 $2^{+/-}$hiPSCs (Fig. 4b). In both cases, the segments looked anatomically normal and were sequentially connected, therefore indicating continuous nephrons (Fig. 4b). In the case of $T S C 2^{-/-}$cell aggregates, the cystic structures stained positive for both the CDH1 and LTL, indicating the cyst-like structures could comprise distal tubule and/or proximal tubule cells (Fig. 4c). Cyst formation occurred with a frequency of $\sim 4.7$ cysts/well for TSC2 $2^{-1-}$ cultures, compared with 0 and $\sim 0.5$ cysts/well for $T S C 2^{+/+}$and $T S C 2^{+/-}$cultures, respectively (Fig. 4b). Of note, $2 \mathrm{D}$ cyst-like structures developed despite the fact that PKD1 expression without addition of cAMP modulators, such as forskolin (Fig. 4h), a method previously employed for the induction of cysts in kidney organoid models of polycystic kidney disease $(\mathrm{PKD})^{34}$. Another observable phenotype in $2 \mathrm{D} \mathrm{TSC2} 2^{-/-}$nephrons was that PODXL1 ${ }^{+}$cells presented a less compact appearance, and were loosely grouped, suggesting dysmorphic glomerular structures (Fig. 4c). These phenotypic characteristics were not associated with changes in the expression of nephron segment markers such as PODXL1, NPHS1, and $S Y N P O$ (glomerulus); AQP1 (proximal tubule); or EPCAM and $\mathrm{CDH1}$ (distal tubule), whose mRNAs were detected at comparable levels in our RNAseq analysis of $T S C 2^{+/+}, T S C 2^{+/-}$and $T S C 2^{-1-}$ organoids (Supplementary Fig. 5a), with exception of
$\mathrm{CDH} 2$ (proximal tubule), which was upregulated in $\mathrm{TSC}^{-1-}$ organoids (Supplementary Fig. 5a).

These observations prompted us to analyze the spatial arrangements of cystic nephron structures in 3D organoids. On Day 18 of the 3D differentiation protocol, we observed protruding dome-like structures indicating incipient cyst formation on the surface of $T S C 2^{-1-}$, but not of TSC2 $2^{+1+}$ or TSC2 $2^{+1-}$ differentiating spheroids by Day 16 (Supplementary Fig. 5b). On Day 21, individual cysts growing out of the $T S C 2^{-1-}$ AML organoids were clearly observed by brightfield microscopy (Fig. 4d). Similar to what has been reported in organoid models of polycystic kidney disease, we found the size of cysts to be larger in 3D aggregates grown on low-attachment surfaces compared to $2 \mathrm{D}$ cell cultures (Supplementary Fig. 5b). Nephron segment analysis using immunofluorescence microscopy confirmed that $\mathrm{TSC}^{+1+}$ and TSC2 $2^{+/}$metanephric mesenchyme aggregates had formed kidney organoids as indicated by the detection of properly segmented nephrons in which $\mathrm{CDH}^{+}$distal tubules were connected with $\mathrm{LTL}^{+}$proximal tubules, and PODXL1 ${ }^{+}$ nephrons, on Day 21 of the differentiation protocol (Fig. 4e). In TSC2 ${ }^{-1-}$ AML organoids, the analysis confirmed that the cells conforming the lining of the cysts stained positive for CDH1 and/ or LTL, but not for PODXL1, indicating that similar to what is observed in TSC patients ${ }^{35}$ the cysts are specifically associated with tubular nephron segments, but can also emerge from multiple segments (Fig. 4f, Supplementary Fig. 5c, d). The immunofluorescence analysis also showed that while mainly individual tubule segments were involved, cysts lined by a combination of both the proximal and distal tubule cellular components were also observed in TSC2 $2^{-1-}$ AML organoid cysts, possibly representing transition regions (Fig. 4f). Unlike the single-cell layers observed lining the cyst regions associated with well-defined tubule segments, the mixed-cell lining regions were multilayered (Fig. 4f), resembling the columnar epithelium that can be observed in the renal cysts of TSC patients ${ }^{35}$. The distribution of LTL staining, representing polarity of brush borders, revealed another aspect of TSC cystic disease, namely loss of cell polarity in the single-cell proximal tubule stretches of cyst lining, in contrast to the polarized cells observed in $\mathrm{TSC}^{+/-}$ tubules (Fig. 4g). Of note, cyst formation in $\mathrm{TSC}^{-/-} \mathrm{AML}$ organoids did not involve loss of polycystin 1 (PKD-1) expression, as indicated by the similar mRNA levels detected in our organoid RNAseq analysis (Fig. 4h).

These findings indicated that $T S C 2^{-/-}$renal organoids recapitulate key features of cystic kidney disease associated with TSC, and support the concept that renal epithelial cyst formation is also driven by loss of TSC2. 


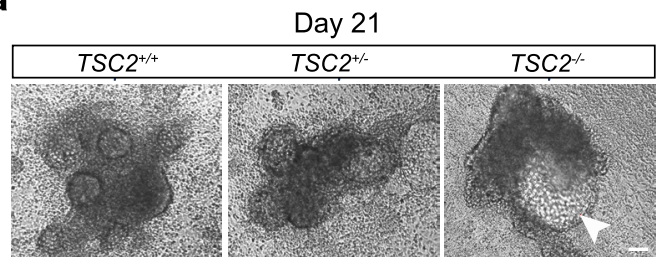

b

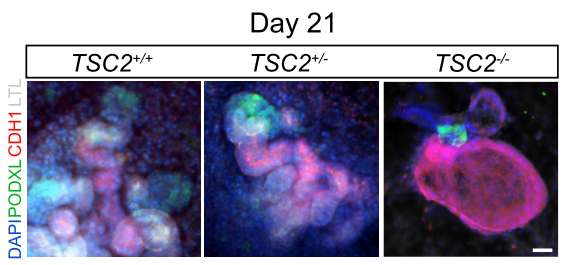

d

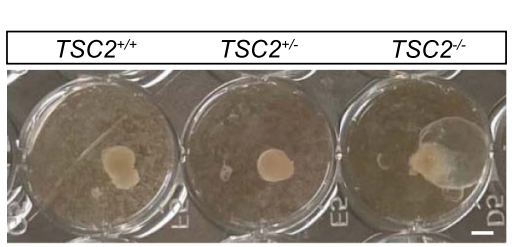

2D cysts

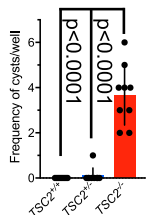

3D cysts c

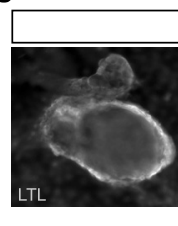

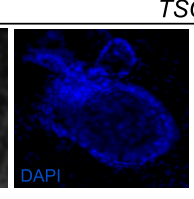
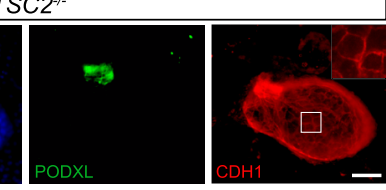

$\mathbf{f}$
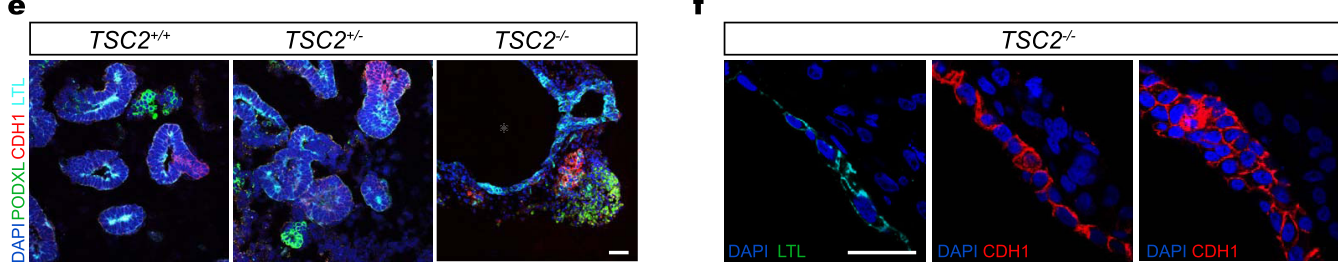

$\mathbf{g}$
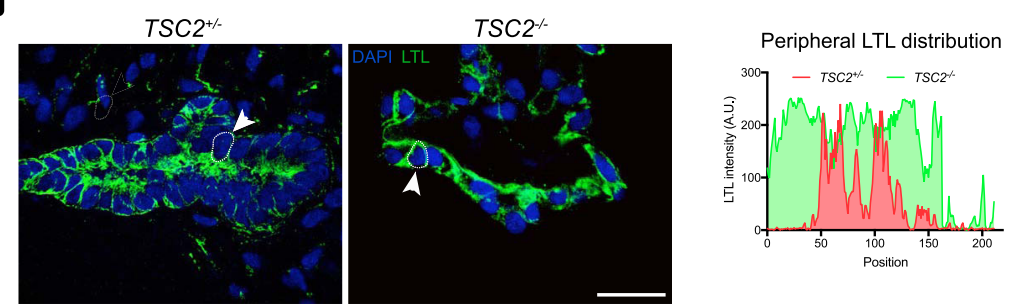

h
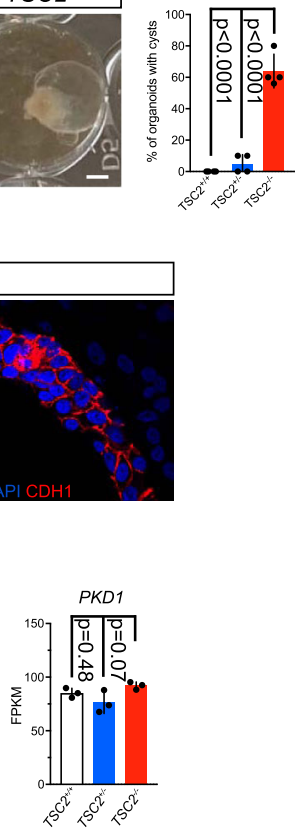

Fig. 4 TSC2 inactivation drives cystogenesis during nephric differentiation. a Representative brightfield images of $2 \mathrm{D}$ TSC2 ${ }^{-/}-$hiPSC-derived renal tissues on Day 21 of differentiation. The arrowhead indicates a visible cyst. Twenty independent experiments were performed with similar results. b Immunofluorescence image showing 2D nephrons derived from the three hiPSC genotypes, labeled with PODXL1 (glomerulus), LTL (proximal tubule), and $\mathrm{CDH} 1$ (distal tubule). Number of cysts per well was quantified. Bar graphs represent mean \pm SD, $n=8$ wells from 2 independent experiments. c The cyst framed in the TSC2-/- nephron micrograph in (a) is stained for nephron markers by immunofluorescence with inset showing the distal tubule epithelium. Ten independent experiments were performed with similar results. d Brightfield image of 3D organoids of the three genotypes showing cystic $\mathrm{TSC}^{-/}$- organoids, with quantification of cyst formation. Bar graphs represent mean \pm SD, $n=4$ experiments. e Representative confocal sections showing organoid glomeruli, proximal and distal tubule regions as indicated by PODXL1, LTL, and CDH1 staining. A cyst associated with a proximal tubule is shown

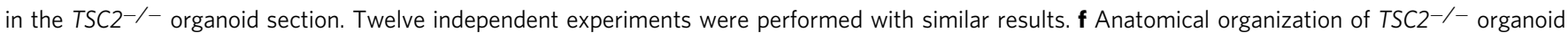
cyst lining visualized using confocal imaging, both proximal tubule epithelial cell (PTEC) and distal tubule epithelial cell (DTEC) single-cell layers and DTEC multicellular regions are shown with $\mathrm{LTL}$ and $\mathrm{CDH} 1$ immunofluorescence. Eight independent experiments were performed with similar results.

g Representative confocal imaging showing the alterations in polarity observed in PTECs lining TSC2 $^{-/-}$organoid cysts, compared to TSC2 ${ }^{+} /-$organoid

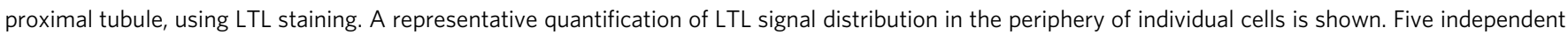
experiments were performed with similar results. h Expression levels for PKD1 in TSC2 ${ }^{-} /{ }^{-}$TSC2 $+/+$, and TSC2 $+/-$renal organoids. Bar graphs represent mean \pm SD, $n=3$ samples for each genotype, five organoids per sample. Gene expression is shown in FPKM values. $P$ values for individual comparisons done using Tukey test in one-way ANOVA analysis are indicated. Scale bars, $50 \mu \mathrm{m}(\mathbf{a}, \mathbf{b}, \mathbf{c}, \mathbf{e}), 25 \mu \mathrm{m}(\mathbf{f}, \mathbf{g})$, and $1 \mathrm{~mm}(\mathbf{d})$.

Transplanted $T S C 2^{-1-}$ AML organoids recapitulate TSCassociated kidney AML and cystic disease in vivo. After determining that our in vitro TSC2 $2^{-1-}$ hiPSC-derived renal organoids recapitulated major features of AMLs TSC-associated cystic disease, we sought to investigate the effect of vascularization on phenotype development. The paucity of vascularization is a major limitation of in vitro hPSC-derived kidney organoid models ${ }^{36}$. We transplanted Day-18 TSC2 ${ }^{-1-}$ pre-organoid stage spheroids into the kidneys of 10 -week-old immunodeficient RNU rats (Fig. 5a, b). We sought to establish how in vivo vascularization alters the anatomy and growth of $T S C 2^{-1-}$ AML organoids, and whether this approach would result in an animal model capable of recapitulating TSC-associated renal abnormalities. Our strategy took advantage of the size of RNU rat kidneys, which allowed for the simultaneous transplantation of two $\sim 500 \mu \mathrm{m}$ Day-18 spheroids (Fig. 5a, b). In addition, we transplanted organoids into both kidneys of each rat, giving us the ability to compare xenografts with different genotypes in the same animal.

Assessment of xenograft size indicated a significantly higher growth rate for $T S C 2^{-1-}$ AML organoids (4.43 fold change), compared to $T S C 2^{+/-}$(3.55 fold change) or $T S C 2^{+/+}$kidney organoids (3.4 fold change) at Day 14 post-transplantation (Fig. 5c). At Day 14, cysts could be seen on the surface of kidneys containing TSC2-1- AML organoids but not in kidneys carrying the $\mathrm{TSC}^{+/-}$or $\mathrm{TSC} 2^{+/+}$organoids (Fig. $4 \mathrm{~d}$ ). At this time point, we surgically removed the rat kidneys and examined the xenografts. To distinguish human organoid tissues from rat tissues by means of immunofluorescence microscopy, we used 
a

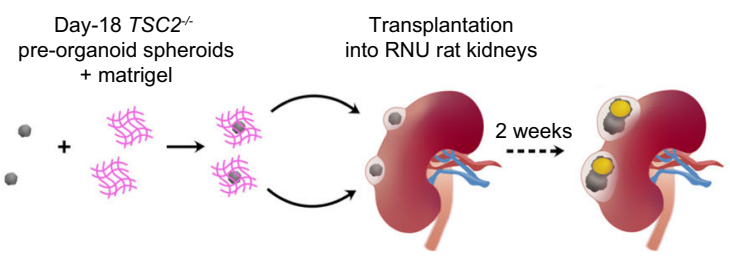

b

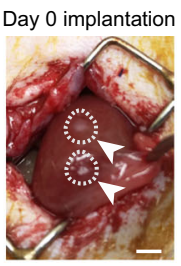

c

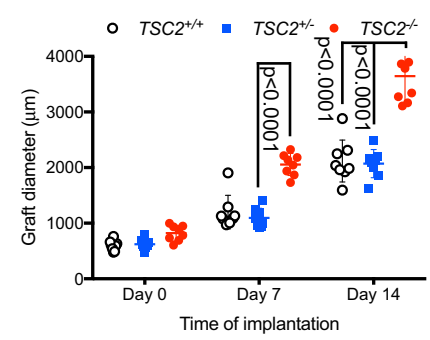

d

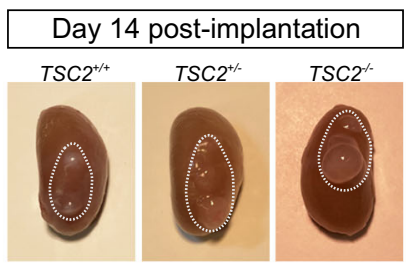

e

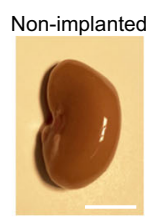

Day $14 \mathrm{TSC2}^{-/} \mathrm{AML}$ organoid xenografts

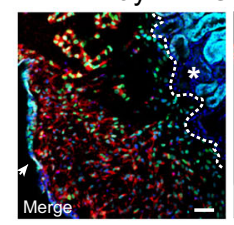

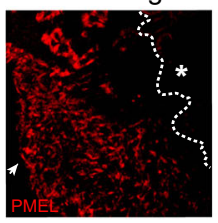

$\mathbf{f}$

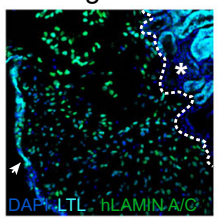

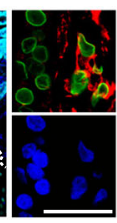

f $T S C 2^{-/}$graft glomerulus

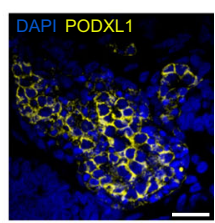

$\mathbf{g}$

Day $14 T_{S C 2}-$ AML xenograft vascularization
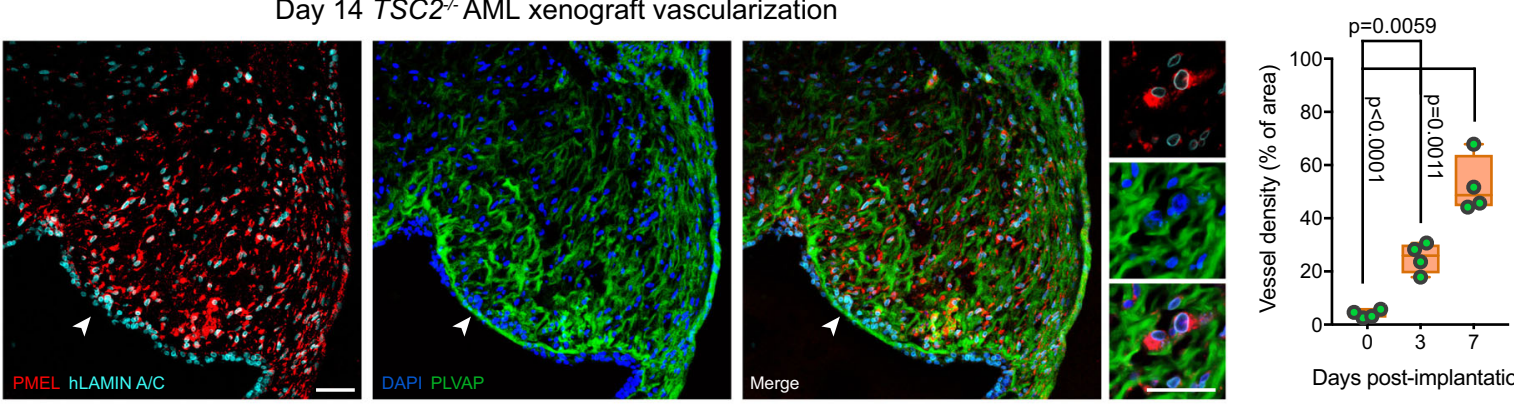

Days post-implantation

i mTOR signaling in AML cells

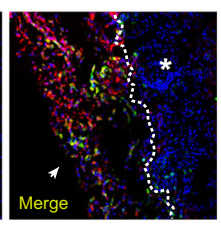

mTOR signaling in cyst lining cells
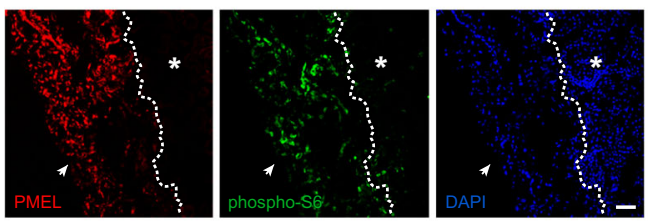

Day 7 AML cell proliferation
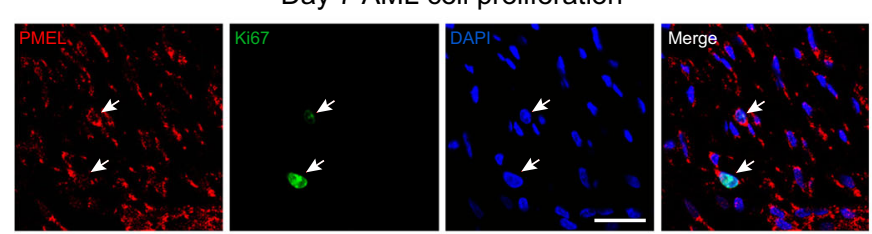

$\mathbf{k}$

Day 7 cyst TEC proliferation
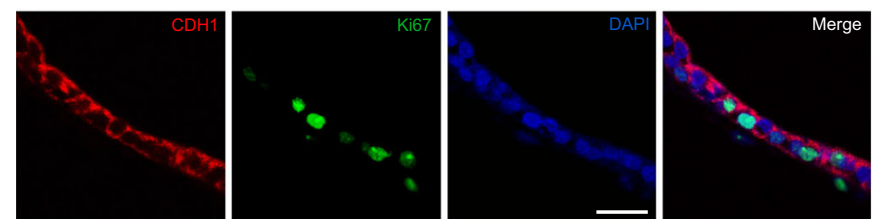
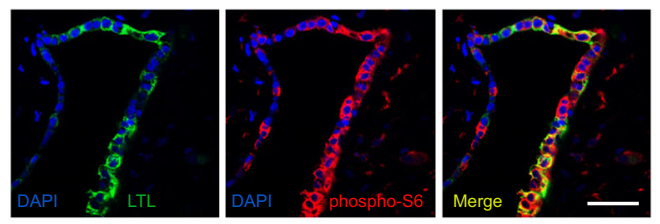

I

Cell proliferation

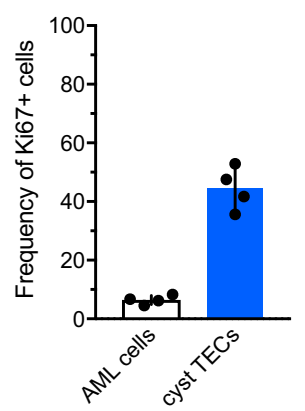

antibodies against either the human nuclear antigen (HNA) or the human isoform of Lamin A/C (hLamin A/C). Analysis of AML markers revealed the widespread and abundant presence of human cells expressing either ACTA2 or PMEL in the TSC2-1xenografts, but not in TSC2 $2^{+/-}$or $T S C 2^{+/+}$organoid xenografts, indicating that the AML phenotype was maintained after engraftment (Fig. 5e). In addition to AML-like cells, nephron tubule epithelium (Fig. 5e, arrowhead) and glomeruli (Fig. 5f) were also preserved in the transplanted organoids. Of note, both the $\mathrm{ACTA} 2^{+}$and $\mathrm{PMEL}^{+}$cells were observed in close proximity to microvascular endothelium of host origin that was visualized through detection of the marker plasmalemma vesicle-associated protein (PLVAP) (Fig. 5g), which is expressed by the microvasculature of the kidney ${ }^{37}$. In addition, mTOR activation, assessed by pS6 expression, was observed in both ACTA2 ${ }^{+}$or $\mathrm{PMEL}^{+}$cells of TSC2 ${ }^{-/-}$AML organoid graft cells, but not in the 
Fig. 5 Orthotopic TSC2 ${ }^{-/-}$renal organoid xenografts model TSC-associated AML and TSC cystic disease in vivo. a Schematic depicting the strategy used for the transplantation of Day 18 pre-organoid spheroids in the subcapsular region of RNU rat kidneys. $\mathbf{b}$ Representative image showing two spheroids implanted on Day 0, each indicated by an arrowhead. c Quantification of graft size over the period of two weeks. Scatter dot plot shows mean \pm SD, $n=8$ grafts, 4 rat kidneys. $P$ values determined by two-way ANOVA analysis using Tukey test for multiple comparisons are indicated. $\mathbf{d}$ Representative image of RNU rat kidneys with engrafted $\mathrm{TSC2}^{+/+}, \mathrm{TSC2}^{-/-}$, and $\mathrm{TSC2}^{+/-}$organoids on Day 14 post-transplantation. e Representative confocal sections of $\mathrm{TSC}^{-} /-$AML grafts showing wide distribution of human PMEL + cells and LTL+ PTECs forming the cyst lining, the latter indicated by a white arrow. LTLstained rat proximal tubules are indicated by a white star. Eight independent experiments were performed with similar results. $\mathbf{f}$ Representative confocal image of a glomerulus containing labeled with PODXL1+ cells in a TSC2-/- $\mathrm{AML}$ graft. Eight independent experiments were performed with similar results. g Representative immunostaining and confocal images showing the thorough vascularization indicated by PLVAP+ vessels, observed in close apposition to $\mathrm{PMEL}^{+}$cells. Quantification of vessel density shown in the floating bar graph for grafts harvested on Day 0, Day 3 and Day 7 post-transplantation. Values represent mean $\pm S D, n=4$ grafts. $P$ values for individual comparisons done using Tukey test in one-way ANOVA analysis are indicated. $\mathbf{h}, \mathbf{i}$ Representative confocal immunofluorescence images for phospho-S6 in PMEL ${ }^{+}$cells (h) and cystlining PTECs (i) in TSC2 ${ }^{-/-}$AML xenografts. In (h) the white arrow indicates the luminal compartment, while the white star indicates the rat kidney. Five independent experiments were performed with similar results. j, $\mathbf{k}$ Detection of Ki67 in $\mathrm{PMEL}^{+}$cells (j) and PTECs (k) in TSC2-/- AML grafts on Day 7 post-transplantation. The white arrows indicate Ki67-expressing human $\mathrm{PMEL}^{+}$cells. Five independent experiments were performed with similar results. I Quantification of $\mathrm{PMEL}^{+} \mathrm{Ki}_{67}+\mathrm{and}_{\mathrm{LTL}}+\mathrm{Ki} 7^{+}$cells. $\mathrm{Values}$ in the bar graph represents mean $\pm \mathrm{SD}, n=4$ grafts. Scale bars, $1 \mathrm{~cm}(\mathbf{b}), 2 \mathrm{~cm}(\mathbf{d}), 50 \mu \mathrm{m}(\mathbf{e}, \mathbf{f}, \mathbf{g}, \mathbf{h}, \mathbf{i}), 25 \mu \mathrm{m}(\mathbf{e}, \mathbf{g}$ high magnification panels), $25 \mu \mathrm{m}(\mathbf{i}, \mathbf{j}, \mathbf{k})$.

adjacent normal rat tissue or in $T S C 2^{+/+}$or $T S C 2^{+/-}$organoid xenografts, indicating that metabolic activation was consistent with xenograft growth (Fig. 5h). Analysis of the tubule epithelium in TSC2 $^{-l-}$ xenografts confirmed the formation of cystic tubules that were lined by PTECs and DTECs in varying proportions, arranged into stretches of single-cell lining, that alternated with stretches presenting a multilayered organization, as observed in TSC2 $2^{-1-}$ organoids in vitro (Fig. 4f). Similar to the TECs found in the in vitro cysts of TSC2 $2^{-1-}$ AML organoids, the TECs present in the in graft cysts lacked polarity and had activated mTORC1 signaling. Similar to the AML cells, activation of mTORC1 activation was also observed in epithelial cyst cells of the TSC2 $^{-1-}$ grafts (Fig. 5i). Despite the common activation of mTORC1 observed in both the AML cells and TECs, most proliferative activity occurred in the cysts lining (Fig. 5j, k, l), suggesting that graft growth was mainly a consequence of cyst hyperplasia. This result is consistent with both the lack of proliferative activity observed in classic kidney AML cells ${ }^{38}$, and the neoplastic activity observed in TSC-associated kidney cyst lining epithelial cells ${ }^{35,39}$.

Collectively our in vivo experiments showed that orthotopic transplantation enhanced the phenotype of $T S C 2^{-/-} \mathrm{AML}$ organoids, permitting the recapitulation of human TSCassociated AML in vivo with a high degree of anatomical and molecular fidelity.

Delivery of rapamycin-loaded nanoparticles abrogates orthotopic TSC2 $^{-1-}$ AML xenografts. Our results with transplanted TSC2 ${ }^{-1-}$ AML organoids in RNU rats prompted us to use this animal model to examine the effect of a nanoparticle formulation for delivery of the mTOR inhibitor rapamycin, a compound used for the treatment of AML tumors in TSC patients ${ }^{33}$. To this end, we designed an experiment in which Rapa was encapsulated in ultra-fine solid biopolymeric nanoparticles and delivered into the subcapsular space adjacent to the organoid grafts (Supplementary Fig. 6a-e). We devised this approach to enable targeted delivery of small doses of rapamycin locally over systemic delivery, which can cause undesired drug effects in other organs. We confirmed the absorption of rapamycin-loaded nanoparticles by kidney organoids in vitro, by visualizing the uptake of nanoparticles loaded with Bodipy 650/665-X (Supplementary Fig. 6f). Incubation of kidney organoids with nanoparticles loaded with $500 \mathrm{ng}$ rapamycin, in vitro, resulted in progressive loss of tissue viability within $48 \mathrm{~h}$ (Supplementary Fig. 6g). Based on these results, we tested two doses of rapamycin nanoparticles (Rapa-Nps) for the in vivo treatments, namely $500 \mathrm{ng}$ and $2 \mu \mathrm{g}$, which we injected under the kidney capsule approximately $2 \mathrm{~mm}$ away from each
TSC2 $2^{-1-}$ AML organoid xenograft, 2 weeks after transplantation. We repeated the treatment 3 days later to complete a regimen of two Rapa-Np injections, and harvested the whole kidneys one week after the first injection (Fig. 6a). We measured the size of the organoid xenografts 3 days and 1 week, i.e., at tissue-collection time- after the first injection. Our measurements showed that Rapa-Np treatment resulted in a rapid reduction of organoid xenograft size (Fig. 6b) of cyst size, with only a few scattered cystlining cells containing pyknotic nuclei observed at the end of the treatment (Supplementary Fig. 7a). This result was in contrast with the effect of oral rapamycin, which effectively blocked xenograft growth but did not result in significant shrinkage after 14 days of treatment (Supplementary Fig. 7b). Immunofluorescence staining showing Caspase 3 (CASP3) activation in $\mathrm{PMEL}^{+}$cells of treated xenografts, but not in cells of control nontreated xenografts, suggested that the Rapa-Np treatmentinduced cell apoptosis (Fig. 6c, Supplementary Fig. 7c). Western blotting of organoid xenografts showed increased cleaved caspase 3 at Days 3 and 7 of treatment (Fig. 6e). In addition to the analysis of CASP3 activity, we detected increasing DNA fragmentation in $\mathrm{PMEL}^{+}$cells of treated xenografts by means histological analysis of terminal deoxynucleotidyl transferase dUTP nick end labeling (TUNEL) (Fig. 6e). DNA fragmentation was not observed in control nontreated TSC2 ${ }^{-1-}$ AML organoid xenografts (Fig. 6e, Supplementary Fig. 7d). Both CASP3 activity and DNA fragmentation indicated that delivery of Rapa-Nps was an effective treatment for local ablation of TSC2-1- AML organoid xenografts.

Taken together, these results highlight the TSC2-1- AML organoid xenografts in RNU rats as a powerful preclinical model for testing drugs and therapeutic approaches for the treatment of AMLs.

Rapamycin nanoparticles disrupt the interaction between p21 ${ }^{\mathrm{CIP1}}$ and pro-CASP3 in TSC2 $^{-1-}$ AML organoid xenografts. Current oral rapalog therapies only partially shrink kidney AMLs in TSC patients ${ }^{8,9}$. Whether mechanisms of tumor resistance reduce the efficacy of these treatments remains unknown. To answer this question we looked at the expression of genes involved in tumor resistance and identified $C D K N 1 A$ as a gene overexpressed in both TSC2 ${ }^{-1-}$ AML organoids and kidney AML compared to control organoids and to normal kidney (Fig. 7a). Further analysis using The Cancer Genome Atlas indicated that CDKN1A is significantly upregulated in kidney AML compared to other tumors (Fig. 7b). These results led us to explore a previously described role for CDKN1A as a driver of tumor resistance through stabilization and accumulation of its encoded protein, 


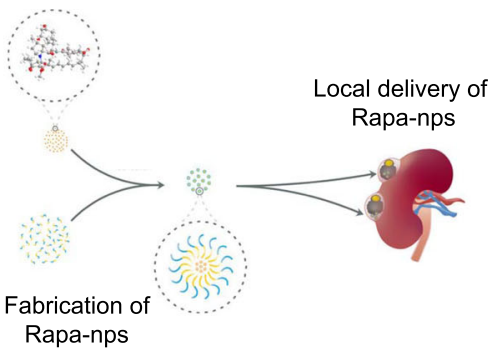

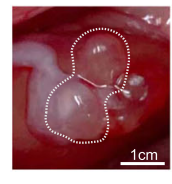
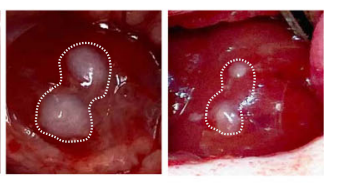

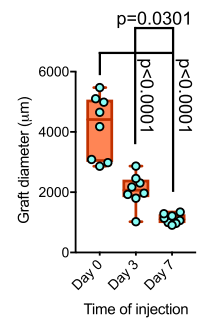

c

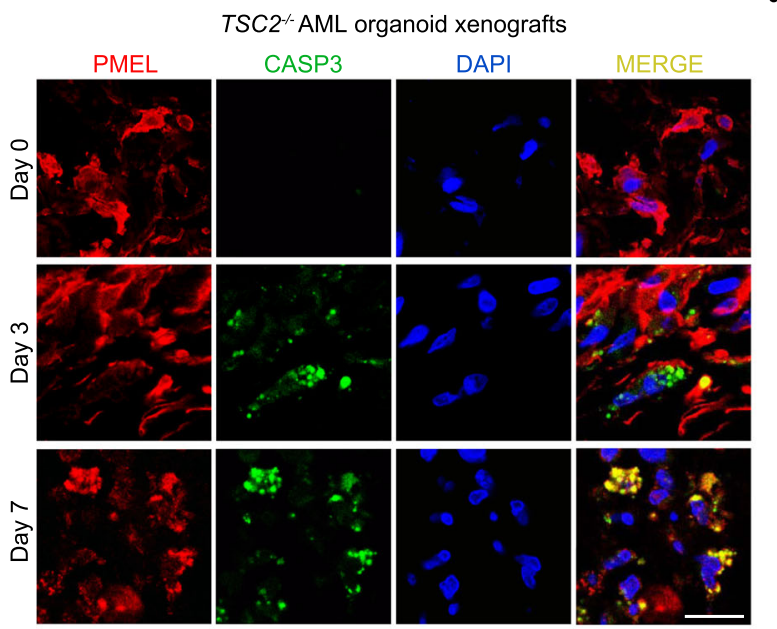

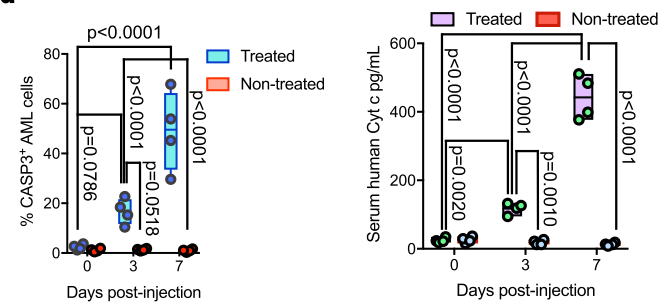

e

Day

post-treatment $0 \quad 3$

CASP3

Cleaved

Cleaved
CASP3

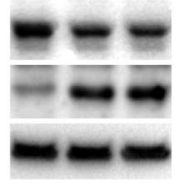

g

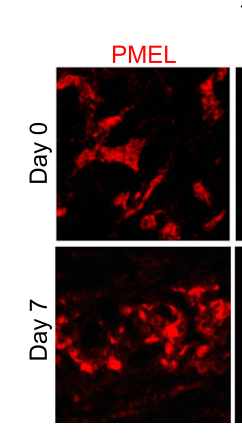

TSC2 ${ }^{-A M L}$ organoid xenografts

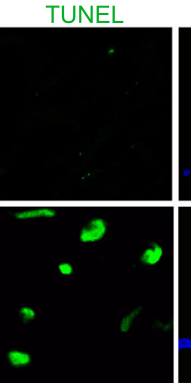

DAPI
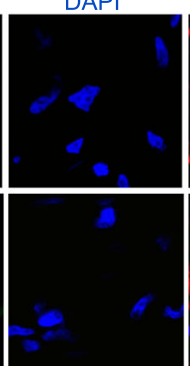

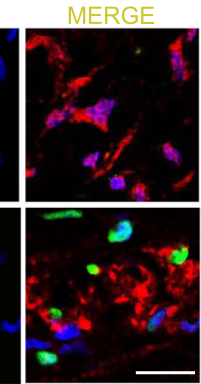

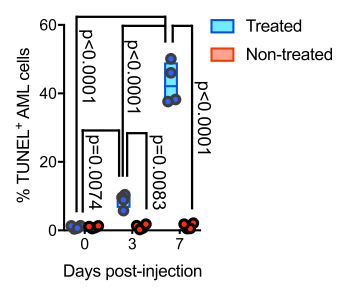

Fig. 6 Ablation of TSC2 ${ }^{-/-}$AML organoid xenografts treated with Rapamycin-loaded nanoparticles. a Schematic showing the strategy for the delivery of Rapa-nanoparticles locally, near the TSC2 ${ }^{-/-}$AML organoid xenografts. b Representative photographs showing the size of TSC2 ${ }^{-/-}$xenografts on Day 3 and Day 7 post Rapa-Np delivery. Quantified diameter values on the floating bar graph represent mean $\pm S D, n=8$ grafts. $P$ values for individual comparisons done using Tukey test in one-way ANOVA analysis are indicated. c Representative immunofluorescence images for the detection of activated Casp3 in TSC2-/- AML organoid xenograft PMEL + myoid cells on Day 3 and Day 7 postdrug delivery. Five independent experiments were performed with similar results. d Floating bar graph with quantification of PMEL ${ }^{+}$Casp3 $3^{+}$cells on Days 3 and 7 postdrug delivery. Mean \pm SD are reported, $n=4$ grafts. $P$ values determined by two-way ANOVA analysis using Tukey test for multiple comparisons are indicated. e Detection of cleaved Casp3 on Day 0 (free organoid), 3 and 7 in protein extracts from TSC2 ${ }^{-/-}$AML xenografts (Day 3 and 7). $f$ Quantification of human Cytochrome $C$ in the serum of RNU carrying rats $T_{S C 2}-/-A M L$ organoid xenografts, treated with Rapa-Np and controls that did not receive the treatment. Floating bar graph shows mean \pm SD, $n=4$ rats per group. $P$ values determined by two-way ANOVA analysis using Tukey test for multiple comparisons are indicated. $\mathbf{g}$ Representative confocal imaging depicting detection of DNA fragmentation by TUNEL on sections of TSC2 ${ }^{-/-}$AML xenografts, three and seven days post Rapa-Np delivery. Five independent experiments were performed with similar results. $\mathbf{h}$ Floating bar graph for the quantification of PMEL + TUNEL + cells shows mean \pm SD are reported, $n=4$ grafts per group. $P$ values determined by two-way ANOVA analysis using Tukey test for multiple comparisons are indicated. Scale bars, $1 \mathrm{~cm}(\mathbf{b}), 25 \mathrm{~mm}(\mathbf{c}, \mathbf{g})$.

namely $\mathrm{p} 21^{\mathrm{CIP} 1}$, in the cell cytoplasm ${ }^{40,41}$. We analyzed $\mathrm{p} 21^{\mathrm{CIP} 1}$ distribution and observed protein accumulation in the cytoplasm of both the TSC2-1- AML organoids and kidney AML (Fig. 7c). This result was consistent with increased accumulation of $\mathrm{p} 21^{\mathrm{CIP} 1}$ in $\mathrm{TSC}^{-1-}$ AML organoid xenografts compared to control
TSC2 ${ }^{+/+}$iPSC-derived xenografts (Fig. 7d), a finding that was concomitant with higher levels of pro-CASP3 in TSC2 ${ }^{-1-}$ AML organoid xenografts (Fig. 7d). Rapa-Np treatment reduced the levels of $\mathrm{p}_{21} \mathrm{CIP}^{\mathrm{P}}$ in treated TSC2 ${ }^{-/}$AML organoid xenografts, compared to nontreated ones, while simultaneously triggering 


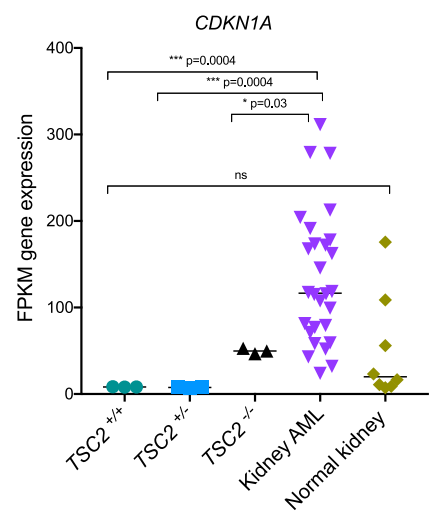

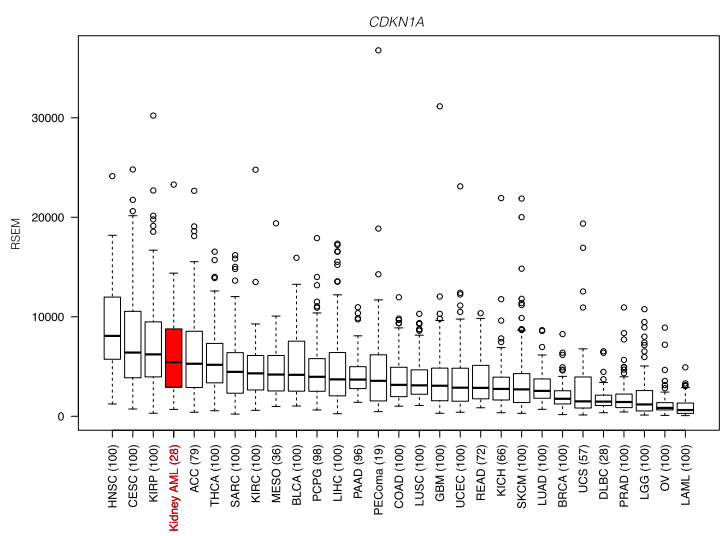
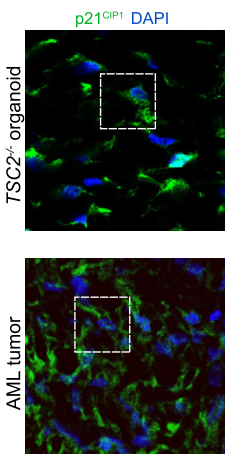
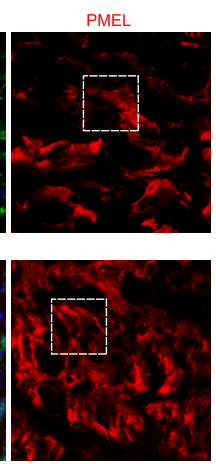
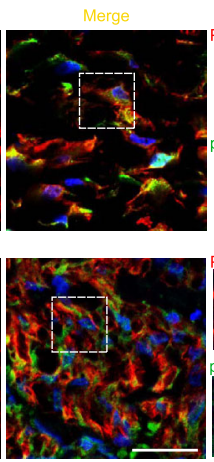

d
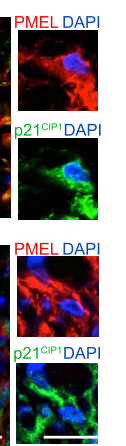

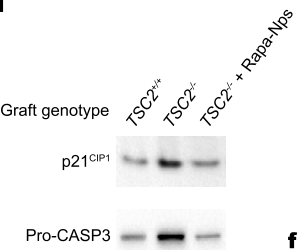

f

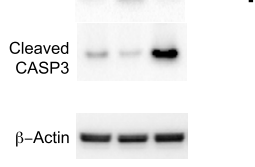

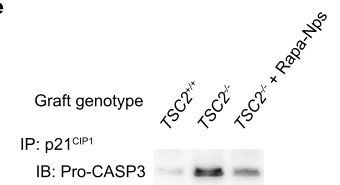

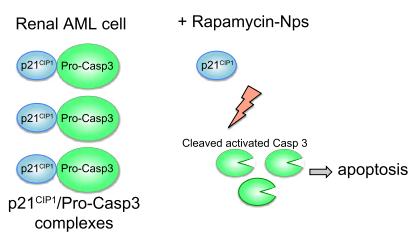

Fig. 7 Rapamycin-Nps disrupt the interaction between p21 $^{\text {CIP1 }}$ and pro-CASP3 in TSC2 ${ }^{-1}$ - organoid AML cells in vivo. a RNAseq data box plots showing expression levels of $C D K N 1 A$ in $T S C 2^{-/-}, \mathrm{TSC}^{+/-}$and $\mathrm{TSC}^{+/}++$renal organoids $(n=3$ each, five organoids per ample), compared to human kidney AML $(n=28)$ and human kidney $(n=8)$ samples. $P$ values for individual comparisons done using a two-sided Mann-Whitney $U$ test are indicated. Gene expression is shown in FPKM values. b Box-and-whisker plot showing minimum value, first quartile, median, third quartile and maximum value for expression of CDKN1A in various tumors using The Cancer Genome Atlas data. Kidney AML is highlighted in red. Sample size for each type of tumor: HNSC $n=100$, CESC $n=100$, KIRP $n=100$, kidney AML $n=28$, ACC $n=79$, THCA $n=100$, SARC $n=100$, KIRC $n=100$, MESO $n=36$, BLCA $n=100$, PCPG $n=98, \mathrm{LIHC} n=100$, PAAD $n=96$, PEComa (19), COAD $n=100$, LUSC $n=100$, GBM $n=100$, UCEC $n=100, \operatorname{READ} n=72, \mathrm{KICH} n=66, \operatorname{SKCM}$ $n=100$, LUAD $n=100$, BRCA $n=100, \operatorname{UCS} n=57, \operatorname{DLBC} n=28, \operatorname{PRAD} n=100$, LGG $n=100$, OV $n=100$, LAML $n=100$. c Representative immunostaining and confocal images showing PMEL and $\mathrm{p} 21^{\mathrm{CIP} 1}$ in TSC2-/- AML organoids and in kidney AML tumor samples. High magnifications show cytoplasmic $\mathrm{p}_{21}{ }^{\mathrm{CIP} 1}$ signal. Three independent experiments were performed with similar results. Scale bars, $25 \mu \mathrm{m}$, and $12.5 \mu \mathrm{m}$ for high magnification panels. d Representative Western Blots showing levels of p21 ${ }^{\mathrm{CIP} 1}$, pro-CASP3 and CASP3 in transplanted $\mathrm{TSC2}^{+/+}$and $\mathrm{TSC2}^{-/-}$organoids. Three independent experiments were performed with similar results. e Representative immunoblots showing co-immunoprecipitated p21 ${ }^{\mathrm{CIP} 1}$ and pro-CASP3 in transplanted $\mathrm{TSC2}^{+/+}$and in $\mathrm{TSC2}^{-/-}$renal organoids untreated or treated with Rapa-Nps. Three independent experiments were performed with similar results.

CASP3 cleavage, by Day 7 (Fig. 7d). The concomitant loss of $\mathrm{p} 21^{\mathrm{CIP} 1}$ and activation of CASP3 prompted us to investigate the prospective interaction between the $\mathrm{p} 21^{\mathrm{CIP} 1}$ and pro-CASP 3 . By means of the co-immunoprecipitation assay we determined that p $21^{\mathrm{CIP} 1}$ directly interacts with pro-CASP3 in $T S C 2^{-1-} \mathrm{AML}$ organoid xenografts, and that this interaction is abrogated by treatment with Rapa-Nps, resulting in accumulation of cleaved CASP3 (Fig. 7e).

Collectively, these data validated our $T S C 2^{-1-}$ iPSC-derived AML organoid xenograft model as an effective tool to investigate mechanisms of AML with implications for the design of more effective therapeutic strategies.

\section{Discussion}

Here we report an experimental strategy for the generation of in vivo models of human renal diseases associated with rare genetic disorders that cannot be reproduced in all their complexity with current experimental animal models. Using a combination of genome editing and hiPSC techniques we show that nephric differentiation of hiPSCs carrying biallelic LOF mutations in the TSC2 locus generates renal tissues that reproduce anatomical and molecular aspects of human myoid AML tumors.

The AML identity of $\mathrm{PMEL}^{+}, \mathrm{ACTA}^{+} \mathrm{TSC} 2^{-/-}$organoid myoid cells is supported by two critical findings, namely the dual smooth muscle and melanocyte cell phenotype, and cell morphology ${ }^{5,6}$. Melanocytes do not express ACTA2, as indicated by single-cell transcriptome profiles of skin cells ${ }^{42,43}$. Similarly, mesenchymal cells in normal kidney tissues do not express melanocyte markers. Both our flow cytometry and histology results show that $\mathrm{ACTA} 2^{+}$mesenchymal cells present in $\mathrm{TSC}^{+/-}$or $\mathrm{TSC}^{+/+}$kidney organoids did not express melanocyte genes, a result that is consistent with single-cell transcriptome profiles of WT kidney organoid mesenchymal cells ${ }^{43}$. Last, in terms of morphology, TSC2 $2^{-/}$organoid AML cells show a distinct plump myoid morphology with that is characteristically observed in AML tumor cells ${ }^{6,44}$, and which clearly differs from the morphology of kidney fibroblasts characterized by elongated spindle-shaped cell bodies ending in prolonged processes, as well as from the multipolar dendritic morphology of skin melanocytes ${ }^{25}$. 
TSC2 ${ }^{-/-}$AML organoids also recapitulated the epithelial cysts observed in TSC patients. Cystic kidney disease is the second most frequent renal manifestation of TSC and can be triggered by the biallelic inactivation of TSC1/TSC2 alone ${ }^{39,45}$ or as the result of deletion events that also affect the PKD1 locus located adjacently in chromosome $16^{46}$. Our RNAseq data showing that levels of PKD1 mRNA in TSC2 ${ }^{-/}$AML organoids were comparable to mRNA levels in $\mathrm{TSC}^{+/-}$and $\mathrm{TSC}^{+/+}$kidney organoids, indicated that the cystogenic mechanisms are solely driven by the loss of TSC2. Cyst formation can also be associated with a subtype of AML called AML containing epithelial cysts (AMLECs) that can be surrounded by condensed subepithelial cambium-like AML cells ${ }^{47,48}$. In the case of AMLECs, the mechanisms of cystogenesis are not well understood, and a role for AML cells, possibly through the secretion of paracrine factors, has been suggested ${ }^{47}$. While those mechanisms have not been investigated in this study, the presence of both an AML phenotype and epithelial cysts is congruent with the idea that early developmental $\mathrm{LOH}$ events affect a broader spectrum of kidney tissues, therefore resulting in multiple RAs ${ }^{14,33}$.

Our experiments in RNU rats represent a major step toward modeling TSC-associated renal manifestations in vivo using transplanted hiPSC-derived kidney organoids. The rat kidney critically provided a vascular bed supporting the growth of $T S C 2^{-}-$organoids, promoting the hyperproliferative activity of cyst lining epithelial cysts, consistent with the clinical notion that factors present in the kidney and the blood play an important role in the development of cystic kidney disease. This in vivo organoid strategy can increase the throughput of disease modeling studies by supporting multiple organoid xenografts that can be used for drug-testing and for mechanistic interrogation. We combined in vitro and in vivo experimental settings to identify overexpression of CDKN1A and direct interaction between its encoded protein $\mathrm{p} 21^{\mathrm{CIP} 1}$ and pro-CASP 3 as a mechanism that can be overridden by nanocarrier-based strategies designed to increase rapalog bioavailability at the site of the lesions. These results are consistent with a previously reported role for $\mathrm{p} 21^{\mathrm{CIP} 1}$ in tumor resistance ${ }^{40,41}$, and with the observation that in certain tumors, accumulation $\mathrm{p} 21^{\mathrm{CIP} 1}$ in the cytoplasm is associated with tumor survival and reduced tumor sensitivity to treatment ${ }^{49-54}$. These findings will be relevant for the design of oral nanotherapies with increased antitumor efficacy. To conclude, we expect that preclinical animal models carrying transplanted hiPSC-derived organoids may become a widely used experimental platform for the study of disease mechanisms and for the development of therapies against molecular targets in human kidney tissues.

\section{Methods}

hiPSC line maintenance. The hiPSC cell lines 77-patient, 77-TSC2-null, and control 77-TSC2WT were a kind gift from Dr. Sahin. The 77-patient line carrying a heterozygous 9-bp deletion in the TSC2 locus was derived from the lymphoblasts of a patient with TSC using the sendai-virus method for the delivery of OCT3/4, KLF4, L-MYC, SOX2, and LIN28 into the cells ${ }^{19}$. The 77-TSC2-null line was generated by introducing a second microdeletion of 6-bp in the WT allele of the 77-patient line, using transcription activator-like effector nucleases TALEN ${ }^{19}$. The 77-TSC2WT line was created by correcting the heterozygous microdeletion of TSC2 in 77-patient cell line with CRISPR-cas9 method ${ }^{19}$. The three hiPSC lines were maintained in $\mathrm{mTeSR}^{\mathrm{mw}} 1$ medium (STEMCELL Technologies \# 85850), in 6-well tissue culture plates (Falcon, \#353046) coated with 1\% vol/vol LDEV-Free hESC-qualified Matrigel (Corning Life Sciences \# 354277) in a $37^{\circ} \mathrm{C}$ incubator with $5 \%$ CO2. Cells were passaged at a 1:3 split ratio once a week, using Gentle Cell Dissociation Reagent (GCDR, STEMCELL Technologies \#07174). Each cell line was maintained below passage 45 and mycoplasma contamination was not detected. Permission for the use of hiPSC lines was granted to DRL by our institutional review board (IRB) at Brigham and Women's Hospital, through IBC protocol 2020B000020.

hiPSC differentiation. hiPSCs maintenance cultures were washed once with PBS (Life Technologies, \#10010-049) and dissociated into single-cell suspensions using
Accutase (STEMCELL Technologies, \#07920). One hundred and fifty thousand cells were plated onto 24 -well tissue culture plates (TPP, \#92024) coated with $1 \%$ Matrigel in $\mathrm{mTeSR}^{\mathrm{mi}} 1$ medium supplemented with ROCK inhibitor molecule

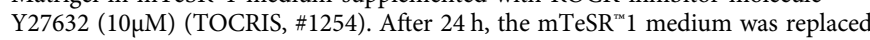
by basic differentiation medium consisting of Advanced RPMI 1640 (Life Technologies, \#12633-020) containing 1X L-GlutaMAX (Life Technologies, \#35050061) supplemented with $8 \mu$ M CHIR99021 (Sigma-Aldrich, \#SML1046) for 4 days. Cells were then cultured in Advanced RPMI + 1X L-GlutaMAX + Activin A $(10 \mathrm{ng} / \mathrm{ml})$ (R\&D, \#338-AC-050) for 3 days. For induction of metanephric mesenchyme the media was replaced with Advanced RPMI + 1X L-GlutaMAX + FGF9 $(20 \mathrm{ng} / \mathrm{ml})(\mathrm{R} \& \mathrm{D}, \# 273-\mathrm{F} 9-025 / \mathrm{CF})$ for 7 days, with a 1 -h pulse of $3 \mu \mathrm{M}$ CHIR added on day 9. To generate 3D organoids, the cells were lifted using Accutase on Day 9 and the cell suspensions incubated with CHIR pulse medium $37^{\circ} \mathrm{C}, 5 \% \mathrm{CO}_{2}$ for $1 \mathrm{~h}$ then switched to basic differentiation medium + FGF9 and plated at 50,000 cells per well onto ultra-low-attachment plates (Corning, \#7007). The plates were centrifuged at 1,500 r.p.m. for $15 \mathrm{~s}$, and the cells then cultured $37^{\circ} \mathrm{C}, 5 \% \mathrm{CO}_{2}$ until day 13 . At day 13 , a pulse of $1 \mu \mathrm{M}$ retinoic acid was added to the medium containing FGF9, and the organoid or cell cultures were incubated for $24 \mathrm{~h}$. At day 14, the cultures where transferred to basic differentiation medium with no additional factors for 7-14 days, and harvested at day 21-28. The differentiation reagents and procedures can be accessed via Protocol Exchange (https:// protocolexchange.researchsquare.com), using DOI: 10.21203/rs.3.pex-1648/v1.

Induction of organoid fibrosis with IL-1ק. Day-21 $\mathrm{TSC}^{+/+}$organoids were incubated in Advanced RPMI + 1X L-GlutaMAX containing $50 \mathrm{ng} / \mathrm{ml}$ human IL1$\beta$ (Sigma-Aldrich \# H6291) for $96 \mathrm{hs}^{29}$. The medium containing IL1- $\beta$ was replaced every $48 \mathrm{~h}$.

Quantitative RT-PCR. Total RNA was extracted using the RNeasy Plus MiniKit (Qiagen). Purity was determined byA260-A280. cDNA was synthesized using oligo(dT) and random primers (iScript Reverse Transcription Supermix; Biorad). Quantitative PCR was performed using the QuantStudio 7 Flex Real-Time PCR System (ThermoFisher Scientific) using TaqMan Gene Expression Assays (ThermoFisher Scientific \#4331182). The specific primer pairs used in quantitative PCR are listed in Supplemental Table 1.

Western blot. In vitro and grafted organoids were lysed in RIPA buffer containing Halt Protease and Phosphatase Inhibitor Cocktail (Thermo FisherScientific), using a glass Dounce homogenizer (DWK Life Sciences Kimble ${ }^{\mathrm{TN}}$ ) on ice. Following protein quantification, SDS-PAGE gel run and western blotting were performed. The following antibodies from Cell Signaling Technology were used: antiribosomal protein S6 (\#2217, clone 5G10, 1:1000), antiphospho S6 (\#2211, 1:1000), antiphospho P70S6K (\#9205, 1:1000), anti-P70S6K (\#9202, 1:2000), anti- $\beta$ Actin (\#4967, 1:1000), anti-GPNMB (\#38313, clone E4D7P, 1:700). Anti-ACTA2 was from Sigma-Aldrich (\#F3777, clone 1A4, 1:2000), antihuman pro-Caspase 3 was purchased from ThermoFisher Scientific (\#MA1-41163, clone 31A893, 1:400), antihuman cleaved Caspase 3 was purchased from Abcam (ab2302, 1:200), antihuman p21CIP1 was purchased from Novus Biologicals (\#AF1047, 1:400). Primary antibodies were detected with peroxidase-conjugated antirabbit or anti-mouse IgG (1:3000) and visualized with SuperSignal West Femto Substrate (ThermoFisher Scientific \#34094). Blots were quantified using ImageJ v1.51 W (https:// imagej.nih.gov/ij/).

Immunofluorescence. Organoids and grafts were fixed with $4 \%$ paraformaldehyde in PBS for $30 \mathrm{~min}$ in a 96-well plate, washed three times in PBS, then incubated with $30 \%$ sucrose $(\mathrm{w} / \mathrm{w})$ overnight at $4{ }^{\circ} \mathrm{C}$. The next day the tissues were mounted into frozen blocks with O.C.T. compound (Fisher Scientific, \#23-730-571) and were cut into 12- $\mu \mathrm{m}$ sections onto glass slides (Fisher Scientific \#22-037-247). The sections were washed three times for $5 \mathrm{~min}$ in PBS, then incubated in blocking buffer containing $0.15 \%$ Triton X-100 and 5\% normal donkey serum) for $1 \mathrm{~h}$, followed by incubation with primary antibodies in antibody dilution buffer $(0.15 \%$ Triton X-100 and $1 \%$ BSA in PBS) for $2 \mathrm{~h}$ or overnight, then washed three times in PBS. Incubation with secondary antibodies in antibody dilution buffer was performed for $1 \mathrm{~h}$, followed by three washes in PBS and coverslips mounted on Vectashield with DAPI (Vector Labs \#H-1200). The primary antibodies used were: anti-SIX2 (Proteintech, \#11562-1-AP, 1:250), anti-SALL1 (Abcam \#ab41974, clone K9814, 1:100), anti-PAX8 (Proteintech, \#10336-1-AP, 1:250), anti-PMEL (Agilent Technologies, \#M063429-2, clone HMB-45, 1:200), anti-ACTA2 (Sigma-Aldrich \#C6198, clone 1A4, 1:400), anti-GPNMB (Cell Signaling Technologies \#38313, clone E4D7P, 1:300), anti-PODXL1 (R\&D Systems, \#AF1658, 1:400), anti-CDH1 (Abcam \# AB40772, clone EP700Y, 1:400), antihuman Lamin A/C (Abcam, \#ab108595, clone EPR4100, 1:300), anti-PLVAP (Univ. of Iowa, MECA32, clone SP2/0, 1:200), antiphospho S6 (CST \#2211, 1:400), anti-Ki67 (ThermoFisher Scientific, \# 701198, clone 12H15 L5, 1:250), antihuman cleaved Caspase 3 (Cell Signaling Technology \#9661, 1:300). Biotinylated lotus tetranoglobus lectin was purchased from Fisher Scientific (\#NC0370187, 1:400) and visualized with streptavidin-Cy5 (ThermoFisher Scientific, \#SA1011, 1:700). Images were taken using a Nikon $\mathrm{C} 1$ confocal microscope. 
Fabrication of rapamycin nanoparticles and gavage delivery. Rapamycin was purchased from LC Laboratories (\# R5000), Poly(ethylene glycol)-block-poly( $\varepsilon$ caprolactone) methyl ether (PEG-PCL, PCL average Mn 5000, PEG average Mn 5000) and Tetrahydrofuran (THF, $\geq 99.9 \%$ ) were acquired from Sigma-Aldrich. Bodipy (FL and 650/665-X, SE) were obtained from Invitrogen. A solvent evaporation method under ultrasonication was used for the encapsulation of rapamycin with PEG-PCL micelles. A 20:1 theoretical loading ratio was used for the system fabrication (10 mg of PEG-PCL diblock copolymer and $0.5 \mathrm{mg}$ of rapamycin). Rapamycin and PEG-PCL were dissolved in tetrahydrofuran and added drop by drop into MillQ water under ultrasonication (Sonic Dismembrator Model 500, Fisher Scientific, Waltham, MA) at 20\% amplification. The solvent was left to evaporate overnight, after which loaded micelles nanoparticles were filtered through a $0.2 \mathrm{um}$ syringe filter to remove non-encapsulated aggregates in the solution. The solution was then centrifuged for $20 \mathrm{~min}$ at a rotational speed of $1150 \times g$ at room temperature. The preparation was stored at $4{ }^{\circ} \mathrm{C}$ to avoid degradation. Nanoparticle morphology and size was analyzed using a JEOL 2010 Advanced High-Performance Transmission Electron Microscope (JEOL, MIT MRSEC, MA). The hydrodynamic radius and Zeta potential for the fabricated rapamycin-loaded nanoparticles were determined by photon correlation spectroscopy using a Zetasizer Nano ZS (Malvern Instruments, Worcester-shire, UK). The hydrodynamic radius was measured using a polystyrene cuvette, and the Zeta potential using a folded capillary Zeta cell. Hydrodynamic radius is reported in $\mathrm{nm}$ and Zeta potential in $\mathrm{mV}$. Chemical analysis of functional groups of the nanoparticles was done using an FTIR spectrophotometer. Measurements for the infrared spectra of the nanoparticles were studied comparing the spectra of the reactants before and after the fabrication to observe the change in chemical composition. Daily oral delivery of rapamycin was done by gavage, at a concentration of $0.5 \mathrm{mg} / \mathrm{Kg}$ in DMSO.

Organoid transplantation and nanoparticle delivery. Ten-week-old male NIHFoxn $1^{\text {rnu }}$ immunodeficient nude rats were purchased from Charles River Laboratories and maintained in the animal unit located at the Harvard Institutes of Medicine. For the surgery, the animals were anesthetized using isoflurane and weighed. The kidney was reached through a $2-\mathrm{cm}$ retroperitoneal incision, and two 18-day organoids embedded in LDEV-Free hESC-qualified Matrigel were placed in the subcapsular space through a 2-mm incision in the kidney capsule. After the dorsal incision was sutured, the animals received $1 \mathrm{ml}$ saline and allowed to recover. Two weeks after organoid transplantation, $2 \mu \mathrm{l}$ of rapamycin-loaded nanoparticle suspensions were mixed with $10 \mu \mathrm{l}$ Matrigel and injected under the kidney capsule, approximately $2-\mathrm{mm}$ away fom each organoid graft. All experiments were performed under animal protocol 2018N00066, approved by the Institutional Animal Care and Use Committee at Brigham and Women's Hospital. We have complied with all relevant ethical regulations for animal testing and research.

Flow cytometry. In each experiment, five organoids of each genotype were incubated with TrypLE Select (Thermo Fisher Scientific \#12563011) in a shaker at $37^{\circ} \mathrm{C}$ for $10 \mathrm{~min}$. The digestions were passed through a $40-\mu \mathrm{m}$ cell strainer (Millipore Sigma \#CLS431750), and washed with PBS. Resulting single-cell suspensions were collected by centrifugation at $400 \mathrm{~g}$ for $5 \mathrm{~min}$. Intracellular stainings with antiPMEL antibody HMB-45-FITC (Novus Biologicals \#NBP2-34638F, 1:200) and with anti-ACTA2 (Sigma-Aldrich \#C6198, 1:400) were performed using the PerFix-nc Kit (Beckman Coulter \#B31167), for 30 min at $4{ }^{\circ} \mathrm{C}$ in supplemented PBS containing $2 \mathrm{mM}$ EDTA and 2\% FBS. Staining for human GPNMB (Fisher Scientific \#17-983-842) was done on nonfixed live cell suspensions for $30 \mathrm{~min}$ at $4{ }^{\circ} \mathrm{C}$ in supplemented PBS containing $2 \mathrm{mM}$ EDTA and 2\% FBS. Analysis was performed on LSRII (Becton Dickinson) equipped with three lasers. Data were collected using FacsDIVA v4.1 software. Sorting gates were defined on the basis of fluorescence-minus-one control stains, using FlowJo 10.4.1.

EdU TUNEL imaging. TUNEL imaging was performed on frozen fixed graft tissue sections using the Click-iT TUNEL Alexa Fluor Imaging Assay (Invitrogen), following the manufacturer's instructions. Briefly, the sections were incubated with anti-PMEL antibody for $1 \mathrm{~h}$ at room temperature, then washed and fixed with $4 \%$ PFA for $15 \mathrm{~min}$ at room temperature, followed by permeabilization with $0.25 \%$ Triton X-100 in PBS for 20 min at room temperature. The sections were then incubated for $1 \mathrm{~h}$ at $37^{\circ} \mathrm{C}$ with labeling reaction mix containing TdT and nucleotide mix including EdUTP. The sections were washed and incubated in Click reaction mix including the Alexa Fluor 488 azide. After washing, the slides were incubated with Alexa 546 anti-mouse secondary antibody and stained with DAPI.

Whole organoid transcriptome RNA sequencing and bioinformatics analysis. For each sample, total RNA was extracted from 5 organoids using the RNeasy Plus MiniKit (Qiagen). Purity was determined byA260-A280. Copy DNA (cDNA) library preparation and sequencing was performed at the Molecular Biology Core Facilities at Dan- Farber Cancer Institute. Samples were sequenced using an Illumina MiSeq sequencer and paired-end 100-bp reads generating 20-30 M reads per sample library. Raw Illumina output was converted to Fastq format using Illumina Bcl2fastq v2.18. The hg19 reference sequence was used for sample alignment.
DESeq2 differential gene expression analysis was done using Qlucore v3.6. Hallmark, KEGG and Gene Ontology (GO) analyses for organoid comparisons were performed using Gene Set Enrichment Analysis tool (GSEA; https://www.gseamsigdb.org) using a phenotype permutation of 1000 . The false discovery rate (FDR) used to define overrepresented gene sets was $q<0.25$.

Human kidney angiomyolipoma RNA sequencing. Whole RNA samples were prepared from 18 fresh/frozen kidney AML samples. Copy DNA (cDNA) library preparation and sequencing was performed at the Molecular Biology Core Facilities at Dan- Farber Cancer Institute. Samples were sequenced using an Illumina HiSeq300 sequencer. All samples were discard pathologic specimens derived from patients who underwent surgical tumor resection. The samples were anonymized, no information regarding gender, age, or genotype was available. This set of samples were combined with publicly available data from 10 additional kidney angiomyolipomas and four normal kidneys ${ }^{55}$ for downstream analyses. The protocol 2014P001446 "Discard human pathologic specimens" was approved by the Partners Human Research Committee of the Mass General Brigham hospitals.

Enolase 2 and Cytochrome C ELISA. For the measurement of Cytochorme C, blood samples were collected from the tail vain of RNU rats prior to the transplantation procedure and on days 3 and 7 after surgery. Cytochrome $C$ was detected the serum samples using the Human Cytochrome C ELISA Kit (ab221832) following the manufacturer's instructions. Enolase 2 concentration was measured in whole organoid extracts using the human ENO2 ELISA kit (Proteintech, \#KE00050), following the manufacturer's instructions.

Enolase 2 activity. Enolase 2 activity was measured in whole organoid extracts using the human ENO2 ELISA kit (Proteintech, \#KE00050), following the manufacturer's instructions. During sample preparation, three organoids per sample were homogenized in $100 \mu \mathrm{L}$ of ice-cold Enolase Assay Buffer. The extracts were centrifuged at $10,000 \mathrm{~g}$ for $5 \mathrm{~min}$ to remove insoluble material. Ten microliters of supernatant were transferred to the plate wells for measurement, to which $50 \mu \mathrm{L}$ of the Reaction Mix were added to the wells. After incubating at $25^{\circ} \mathrm{C}$ for $10 \mathrm{~min}$, fluorescence intensity was read at $\lambda_{\text {ex }}=570 \mathrm{~nm}$. Activity was calculated using the formula: Enolase Activity $=\left(\right.$ nmole of $\mathrm{H}_{2} \mathrm{O}_{2} \times$ Sample Dilution Factor $) /($ Reaction Time) $\times$ Volume.

Immunoprecipitation assay. Immunoprecipitation experiments were performed using an amine-reactive resin-based method (Pierce, \#26149), using antihuman p21 CIP1 (Novus Biologicals, \#AF1047, 1:200). Immunoblotting was performed using the antibody antihuman pro-Caspase 3 from ThermoFisher Scientific (\#31A893, 1:300). Primary antibodies were detected with peroxidase-conjugated antirabbit or anti-mouse IgG (1:3000) and visualized with SuperSignal West Femto Substrate (ThermoFisher Scientific \#34094).

Statistics. Student's $t$ test, one-way ANOVA and two-way ANOVA were used. Where applicable, normal distribution of the data was determined using D'Agostino-Pearson and Shapiro-Wilk normality tests. Statistical analysis was performed using Prism 7 for Mac OS (GraphPad Software, Inc.).

Reporting summary. Further information on research design is available in the Nature Research Reporting Summary linked to this article.

\section{Data availability}

The RNASeq datasets are available under GEO series record "GSE171474". Further information and data that support the findings of this study are available within the article and its Supplementary Information files or from the corresponding author upon reasonable request. A Source Data file with the raw results is provided with this paper. Source data are provided with this paper.

Received: 19 March 2021; Accepted: 13 October 2021; Published online: 11 November 2021

\section{References}

1. Bernstein, J. \& Robbins, T. O. Renal involvement in tuberous sclerosis. Ann. N. Y Acad. Sci. 615, 36-49 (1991).

2. Bissler, J. J. \& Christopher Kingswood, J. Renal manifestation of tuberous sclerosis complex. Am. J. Med. Genet. Part C., Semin. Med. Genet. 178, 338-347 (2018)

3. Thway, K. \& Fisher, C. PEComa: morphology and genetics of a complex tumor family. Ann. diagnostic Pathol. 19, 359-368 (2015).

4. Folpe, A. L. \& Kwiatkowski, D. J. Perivascular epithelioid cell neoplasms: pathology and pathogenesis. Hum. Pathol. 41, 1-15 (2010). 
5. Kaiserling, E., Kröber, S., Xiao, J. C. \& Schaumburg-Lever, G Angiomyolipoma of the kidney. Immunoreactivity with HMB-45 Light- and electron-microscopic findings. Histopathol. 25, 41-48 (1994).

6. Stone, C. H. et al. Renal angiomyolipoma: further immunophenotypic characterization of an expanding morphologic spectrum. Arch. Pathol. Lab. Med. 125, 751-758 (2001).

7. Hodges, A. K. et al. Pathological mutations in TSC1 and TSC2 disrupt the interaction between hamartin and tuberin. Hum. Mol. Genet 10, 2899-2905 (2001).

8. Bissler, J. J. et al. Angiomyolipoma rebound tumor growth after discontinuation of everolimus in patients with tuberous sclerosis complex or sporadic lymphangioleiomyomatosis. PLoS One 13, e0201005 (2018).

9. Samuels, J. A. Treatment of renal angiomyolipoma and other hamartomas in patients with tuberous sclerosis complex. Clin. J. Am. Soc. Nephrol. 12, 1196-1202 (2017).

10. Verhave, J. et al. The incidence, management, and evolution of rapamycinrelated side effects in kidney transplant recipients. Clin. Transplant. $\mathbf{2 8}$ 616-622 (2014).

11. Vandewiele, B., Vandecasteele, S. J. \& Vanwalleghem, L. \& De Vriese, A.S. Diffuse alveolar hemorrhage induced by everolimus. Chest 137, 456-459 (2010).

12. El-Hashemite, N., Zhang, H., Henske, E. P. \& Kwiatkowski, D. J. Mutation in TSC2 and activation of mammalian target of rapamycin signalling pathway in renal angiomyolipoma. Lancet (Lond., Engl.) 361, 1348-1349 (2003).

13. Giannikou, K. et al. Whole exome sequencing identifies TSC1/TSC2 biallelic loss as the primary and sufficient driver event for renal angiomyolipoma development. PLoS Genet. 12, e1006242 (2016).

14. Henske, E. P. Tuberous sclerosis and the kidney: from mesenchyme to epithelium, and beyond. Pediatr. Nephrol. 20, 854-857 (2005).

15. Caliò, A. et al. Angiomyolipoma of the kidney: from simple hamartoma to complex tumour. Pathology 53, 129-140 (2021).

16. Du, H. et al. A novel mouse model of hemangiopericytoma due to loss of Tsc2. Hum. Mol. Genet 27, 4169-4175 (2018).

17. Wu, Z. et al. Tsc1 ablation in Prx1 and Osterix lineages causes renal cystogenesis in mouse. Sci. Rep. 9, 837 (2019).

18. Liang, N. et al. Regulation of YAP by mTOR and autophagy reveals a therapeutic target of tuberous sclerosis complex. J. Exp. Med. 211, 2249-2263 (2014).

19. Sundberg, M. et al. Purkinje cells derived from TSC patients display hypoexcitability and synaptic deficits associated with reduced FMRP levels and reversed by rapamycin. Mol. Psychiatry 23, 2167-2183 (2018).

20. Morizane, R. et al. Nephron organoids derived from human pluripotent stem cells model kidney development and injury. Nat. Biotechnol. 33, 1193-1200 (2015).

21. Musah, S. et al. Mature induced-pluripotent-stem-cell-derived human podocytes reconstitute kidney glomerular-capillary-wall function on a chip. Nat. Biomed. Eng. 1, 0069 (2017).

22. Morizane, R. \& Bonventre, J. V. Generation of nephron progenitor cells and kidney organoids from human pluripotent stem cells. Nat. Protoc. 12, 195-207 (2017).

23. Zarei, M. et al. MITF is a driver oncogene and potential therapeutic target in kidney angiomyolipoma tumors through transcriptional regulation of CYR61. Oncogene 40, 112-126 (2021).

24. Roma, A. A., Magi-Galluzzi, C. \& Zhou, M. Differential expression of melanocytic markers in myoid, lipomatous, and vascular components of renal angiomyolipomas. Arch. Pathol. Lab. Med. 131, 122-125 (2007).

25. Hirobe, T. Structure and function of melanocytes: microscopic morphology and cell biology of mouse melanocytes in the epidermis and hair follicle. Histol. Histopathol. 10, 223-237 (1995).

26. Ohta, S. et al. Generation of human melanocytes from induced pluripotent stem cells. PLoS One 6, e16182 (2011)

27. $\mathrm{Wu}, \mathrm{H}$. et al. Comparative analysis and refinement of human PSC-Derived Kidney Organoid Differentiation with Single-Cell Transcriptomics. Cell Stem Cell 23, 869-881.e868 (2018).

28. Siroky, B. J. et al. Evidence for pericyte origin of TSC-associated renal angiomyolipomas and implications for angiotensin receptor inhibition therapy. Am. J. Physiol. Ren. Physiol. 307, F560-F570 (2014).

29. Lemos, D. R. et al. Interleukin-1beta Activates a MYC-Dependent Metabolic Switch in Kidney Stromal Cells Necessary for Progressive Tubulointerstitial Fibrosis. J. Am. Soc. Nephrol. 29, 1690-1705 (2018)

30. Ashfaq, R., Weinberg, A. G. \& Albores-Saavedra, J. Renal angiomyolipomas and HMB-45 reactivity. Cancer 71, 3091-3097 (1993).

31. Cho, J. H. et al. Notch transactivates Rheb to maintain the multipotency of TSC-null cells. Nat. Commun. 8, 1848 (2017).

32. Tapia, F. J. et al. Neuron-specific enolase is produced by neuroendocrine tumours. Lancet (Lond., Engl.) 1, 808-811 (1981).
33. Lam, H. C., Siroky, B. J. \& Henske, E. P. Renal disease in tuberous sclerosis complex: pathogenesis and therapy. Nat. Rev. Nephrol. 14, 704-716 (2018).

34. Freedman, B. S. et al. Modelling kidney disease with CRISPR-mutant kidney organoids derived from human pluripotent epiblast spheroids. Nat. Commun. 6, 8715 (2015).

35. Stapleton, F. B., Johnson, D., Kaplan, G. W. \& Griswold, W. The cystic renal lesion in tuberous sclerosis. J. pediatrics 97, 574-579 (1980).

36. Homan, K. A. et al. Flow-enhanced vascularization and maturation of kidney organoids in vitro. Nat. methods 16, 255-262 (2019).

37. Barry, D. M. et al. Molecular determinants of nephron vascular specialization in the kidney. Nat. Commun. 10, 5705 (2019).

38. Li, W., Guo, L., Bi, X., Ma, J. \& Zheng, S. Immunohistochemistry of p53 and Ki-67 and p53 mutation analysis in renal epithelioid angiomyolipoma. Int. J. Clin. Exp. Pathol. 8, 9446-9451 (2015).

39. Zhou, J., Brugarolas, J. \& Parada, L. F. Loss of Tsc1, but not Pten, in renal tubular cells causes polycystic kidney disease by activating mTORC1. Hum. Mol. Genet 18, 4428-4441 (2009).

40. Cmielová, J. \& Rezáčová, M. p21Cip1/Waf1 protein and its function based on a subcellular localization [corrected]. J. Cell. Biochem. 112, 3502-3506 (2011).

41. Abbas, T. \& Dutta, A. p21 in cancer: intricate networks and multiple activities Nat. Rev. Cancer 9, 400-414 (2009).

42. Ge, W. et al. Single-cell transcriptome profiling reveals dermal and epithelial cell fate decisions during embryonic hair follicle development. Theranostics 10, 7581-7598 (2020)

43. $\mathrm{Wu}, \mathrm{H}$. et al. Comparative analysis and refinement of human PSC-derived kidney organoid differentiation with single-cell transcriptomics. Cell Stem Cell 23, 869-881 e868 (2018).

44. He, W. et al. Epithelioid angiomyolipoma of the kidney: pathological features and clinical outcome in a series of consecutively resected tumors. Mod. Pathol. 26, 1355-1364 (2013).

45. Bissler, J. J. et al. Tuberous sclerosis complex exhibits a new renal cystogenic mechanism. Physiological Rep. 7, e13983 (2019).

46. Sampson, J. R. et al. Renal cystic disease in tuberous sclerosis: role of the polycystic kidney disease 1 gene. Am. J. Hum. Genet 61, 843-851 (1997).

47. Fine, S. W., Reuter, V. E., Epstein, J. I. \& Argani, P. Angiomyolipoma with epithelial cysts (AMLEC): a distinct cystic variant of angiomyolipoma. Am. J. surgical Pathol. 30, 593-599 (2006).

48. Wei, J., Li, Y., Wen, Y., Li, L. \& Zhang, R. Renal angiomyolipoma with epithelial cysts: a rare entity and review of literature. Int. J. Clin. Exp. Pathol. 8, 11760-11765 (2015).

49. Xia, X. et al. Cytoplasmic p21 is a potential predictor for cisplatin sensitivity in ovarian cancer. BMC Cancer 11, 399 (2011).

50. Xia, W. et al. Phosphorylation/cytoplasmic localization of p21Cip1/WAF1 is associated with HER2/neu overexpression and provides a novel combination predictor for poor prognosis in breast cancer patients. Clin. Cancer Res 10, 3815-3824 (2004).

51. Abukhdeir, A. M. \& Park, B. H. P21 and p27: roles in carcinogenesis and drug resistance. Expert Rev. Mol. Med. 10, e19 (2008).

52. Bissonnette, N. \& Hunting, D. J. p21-induced cycle arrest in G1 protects cells from apoptosis induced by UV-irradiation or RNA polymerase II blockage. Oncogene 16, 3461-3469 (1998).

53. Liu, S., Bishop, W. R. \& Liu, M. Differential effects of cell cycle regulatory protein $\mathrm{p} 21$ (WAF1/Cip1) on apoptosis and sensitivity to cancer chemotherapy. Drug Resist Updat. 6, 183-195 (2003).

54. Tian, H., Wittmack, E. K. \& Jorgensen, T. J. p21WAF1/CIP1 antisense therapy radiosensitizes human colon cancer by converting growth arrest to apoptosis. Cancer Res 60, 679-684 (2000).

55. Martin, K. R. et al. The genomic landscape of tuberous sclerosis complex. Nat. Commun. 8, 15816 (2017)

\section{Acknowledgements}

This study was supported by grant funding from the National Institutes of Health (NIH) (R21AG058159 to DRL, R01DK124301 to DRL) and R01NS113591 to MS and the Intellectual and Developmental Disabilities Center at Boston Children's Hospital (BCH IDDRC; U54HD090255).

\section{Author contributions}

J.O.R.H., X.W., M.V.S., M.L.M., M.F.S.R., A.M.H., D.O.L.C., and G.U.R.E. performed experimental studies. M.Su. generated iPSC lines. K.G., and C.K.P. performed data analysis. A.R., E.P.H. and D.J.K., M.Sa., and D.R.L. supervised different aspects of the work. D.R.L. wrote the article.

\section{Competing interests}

The authors declare no competing interests. 


\section{Additional information}

Supplementary information The online version contains supplementary material available at https://doi.org/10.1038/s41467-021-26596-y.

Correspondence and requests for materials should be addressed to D. R. Lemos.

Peer review information Nature Communications thanks Katalin Susztak and the other anonymous reviewer(s) for their contribution to the peer review this work. Peer reviewer reports are available.

Reprints and permission information is available at http://www.nature.com/reprints

Publisher's note Springer Nature remains neutral with regard to jurisdictional claims in published maps and institutional affiliations. (c) (i) Open Access This article is licensed under a Creative Commons Attribution 4.0 International License, which permits use, sharing, adaptation, distribution and reproduction in any medium or format, as long as you give appropriate credit to the original author(s) and the source, provide a link to the Creative Commons license, and indicate if changes were made. The images or other third party material in this article are included in the article's Creative Commons license, unless indicated otherwise in a credit line to the material. If material is not included in the article's Creative Commons license and your intended use is not permitted by statutory regulation or exceeds the permitted use, you will need to obtain permission directly from the copyright holder. To view a copy of this license, visit http://creativecommons.org/ licenses/by/4.0/.

(C) The Author(s) 2021 